\title{
Wall pressure spectra calculations for equilibrium boundary layers
}

\author{
By RONALD L. PANTON
}

Mechanical Engineering Department, The University of Texas, Austin, Texas 78712

\section{AND JOHN H. LINEBARGER}

Western New England College, Springfield, Massachusetts 01095

(Received 30 October 1973)

Assuming information about the mean velocity and vertical turbulent velocity, it is possible to calculate the flow direction wavenumber spectrum of pressure fluctuations $\phi\left(k_{1} \delta\right) / \tau_{0}^{2} \delta$. The law of the wall plus Cole's wake function represented the mean velocity profiles. A scale-anisotropic model of $R_{22}$ was used and the component intensity $\hat{u}_{2}$ was assumed to vary across the boundary layer in constant proportionality to the Reynolds stress. Calculated zero-pressure-gradient spectra rise as $k_{1}^{1 \cdot 5}$ at low wavenumbers. Curves for various Reynolds numbers are closely similar, and diverge only slightly around the peak in the spectrum. A high wavenumber spectrum $\phi\left(k_{1} \nu / u_{*}\right) \cdot u_{*} / \tau_{0}^{2} \nu$ is independent of Reynolds number. The calculations reveal an overlap region in which $\phi \sim k_{1}^{-1}$. Imposing an equilibrium pressure gradient increases the spectrum at the low and mid wavenumbers, but has no effect in the overlap region. The spectrum peak for $\Pi=6$ is a factor $10^{2}$ higher than for the zero-pressure-gradient layer. It is proposed that the convective velocity $U_{c}\left(k_{1}\right)$ has an overlap region. The overlap law is found to be

$$
\frac{U_{c}}{u_{*}}=-\frac{1}{\kappa} \ln k_{1} \delta+\frac{1}{\kappa} \ln \frac{u_{*} \delta}{\nu}+A,
$$

where $\kappa$ and $A$ are the same constants as in the mean velocity expression. Comparison with experiments shows very good agreement. A rough convective 'wake' function is formulated for the low-wavenumber range. Wavenumber spectra are converted to frequency spectra, and compared with experiments. Data from a zero pressure gradient and an adverse pressure gradient $\Pi=3$ show reasonable agreement with the calculations.

\section{Introduction}

Pressure fluctuations on the wall under a turbulent boundary layer result from turbulent fluctuations throughout the layer. In this paper, properties of the pressure field are numerically calculated. Major attention is given to finding $\phi\left(k_{1}\right)$, the spectral density of wall pressure fluctuations, as a function of streamwise wavenumber $k_{1}$. This can be converted to a frequency spectrum by assuming a frozen turbulence field is convected at a velocity $U_{c}\left(k_{1}\right)$ along the wall. 
Early work on the pressure field in isotropic turbulence was done by Batchelor (1951) and Heisenberg (1948). The foundation for our work was laid by Kraichnan $(1956 a, b)$. His papers provide the first investigation of the effects of anisotropy and mean shear on pressure fluctuations. Lilley \& Hodgson (1960), Hodgson (1962) and Lilley (1963), with a different mathematical approach, arrived at a frequency spectrum for a zero-pressure-gradient boundary layer. Their closed form result necessarily included several physical and mathematical approximations. This line of analytical research lay dormant for several years. Our work, which follows Kraichnan's method, involves a five-dimensional integration, which is practical only with modern electronic computers.

The equilibrium boundary layers were assumed to have velocity profiles given by the law of the wall plus Cole's wake function. Extreme pressure gradients are excluded, because we assumed the inner layer $y_{*}<33 \cdot 2$ is not influenced by the pressure gradient. Turbulence assumptions required in the analysis concern $R_{22}$, the vertical velocity correlation coefficient. An isotropic model with an extended scale in the flow direction, scale anisotropy, was used. The integral scale was allowed to be a function of position across the boundary layer. Our hypothesis about the vertical component intensity $\widehat{u}_{2}$ is that it will have similarity for equilibrium boundary layers, and can be related to the Reynolds stress. In fact, we find that $\hat{u}_{2}^{2} \approx \overline{u_{1} u_{2}}$ for all equilibrium layers.

These more detailed assumptions result in spectra that cannot be arbitrarily adjusted to agree with pressure fluctuation experiments. All of the empirical information is obtained from turbulent velocity measurements. The inclusion of variations across the layer is primarily responsible for improving the highfrequency trends over previous theories.

\section{Problem formulation}

The equation which governs pressure fluctuations in turbulence is derived by taking the divergence of the momentum equation, introducing Reynolds decomposition into mean and fluctuating quantities, then subtracting the timeaveraged equation:

$$
\frac{1}{\rho} \frac{\partial^{2} p}{\partial x_{i} \partial x_{i}}=-2 \frac{\partial u_{j}}{\partial x_{i}} \frac{\partial U_{i}}{\partial x_{j}}-\frac{\partial^{2}}{\partial x_{i} \partial x_{j}}\left(u_{i} u_{j}-\overline{u_{i} u_{j}}\right)
$$

(for pressure fluctuation $p$, velocity fluctuation $u_{i}$, and mean velocity $U_{i}$ ). Lower case symbols refer to fluctuation components and upper case symbols to mean values. The terms on the right-hand side are assumed known, and are called the turbulence-mean shear interaction and the turbulence-turbulence interaction. Kraichnan (1956b), Lilley \& Hodgson (1960), Lilley (1964) and Hodgson (1962) estimated the relative importance of these interactions, and concluded that the turbulence-turbulence terms were only 4-6\% of the mean-square value. Hodgson also estimated the spectral distribution of the contribution from the turbulence-turbulence terms. The contribution was always negligible at high and moderate frequencies, but became comparable to the turbulence-mean shear contribution at $\omega \delta_{1} / U_{1}=0.02$ (circular frequency $\omega$, displacement thickness $\delta_{1}$ ), 
roughly $k_{1} \delta=0 \cdot 15$. Corcos (1964) argued that the turbulence-mean shear term is not the only significant contribution (with the exception of high frequencies). The question is one of degree. Below what wavenumber does the turbulence-mean shear term cease to be a major contributor to the spectral density? Our calculations included only the turbulence-mean shear contribution; and on the basis of our own glider experiments (Panton et al. 1971), we believe the computations are valid down to $k_{1} \delta \approx 0 \cdot 5-1 \cdot 0$.

\subsection{Pressure equation solution}

In solving (2.1) for a boundary layer, it is assumed the turbulence is homogeneous in planes parallel to the wall. This means that boundary-layer growth effects are neglected. Far from the wall, the pressure fluctuations are assumed to vanish, while on the wall the normal derivative of the pressure is set to zero. Townsend (1956) derived the wall conditions as an approximation.

We adopted the formulation of the problem given by Kraichnan (1956b). The pressure fluctuations are decomposed into spatial Fourier components in planes parallel to the wall according to

$$
\tilde{p}\left(k_{1}, x_{2}, k_{3}, t\right)=\frac{1}{4 \pi^{2}} \iint_{-\infty}^{\infty} p\left(x_{1}, x_{2}, x_{3}, t\right) \exp \left[-i\left(k_{1} x_{1}+k_{3} x_{3}\right)\right] d x_{1} d x_{3} .
$$

After solution of the transformed differential equation for $\tilde{p}$, the quantity of interest is the spectrum of pressure fluctuations on the wall as a function of wavenumber $k_{1}$. This is obtained by time-averaging the magnitude and integrating over the spanwise wavenumber:

$$
\phi\left(k_{1}\right)=\int_{-\infty}^{\infty} \overline{\tilde{p}\left(k_{1}, 0, k_{3}, t\right) \tilde{p}^{*}\left(k_{1}, 0, k_{3}, t\right)} d k_{3} .
$$

Most of our results are concerned with $\phi\left(k_{1}\right)$. By employing the convection assumption, the $k_{1}$ dependence can be replaced by frequency $\omega$ :

$$
\omega=k_{1} U_{c}
$$

The convective velocity $U_{c}$ may be regarded as a function of $k_{1}$ wavenumber. The spectrum $\Phi(\omega) U_{c}=\phi\left(k_{1} \rightarrow \omega / U_{c}\right)$ represents the frequency spectrum that would be measured at a point on the wall.

Kraichnan's analysis led to the spectrum $\phi(k)$, where $k^{2}=k_{1}^{2}+k_{3}^{2}$. It requires only a slight modification to produce the spectral density in terms of the flow direction wavenumber $k_{1}$.

$$
\phi\left(k_{1}\right)=4 \rho^{2} \int_{-\infty}^{\infty} \int_{\infty}^{\infty} \int_{\infty}^{\infty} \frac{k_{1}^{2}}{k^{2}} \exp \left[-k\left(x_{2}+x_{2}^{\prime}\right)\right] S_{22} \frac{d U_{2}}{d x_{2}}\left(x_{2}\right) \frac{d U_{2}}{d x_{2}}\left(x_{2}^{\prime}\right) d x_{2} d x_{2}^{\prime} d k_{3},
$$

where $k^{2}=k_{1}^{2}+k_{3}^{2}, S_{22}$ is the Fourier transform of the vertical velocity correlation coefficient

$$
\begin{gathered}
R_{22} \equiv \overline{u_{2}\left(x_{1}, x_{2}, x_{3}\right) u_{2}\left(x_{1}+r_{1}, x_{2}^{\prime}, x_{3}+r_{3}\right)} /\left(\hat{u}_{2}\left(x_{2}\right) \hat{u}_{2}\left(x_{2}^{\prime}\right)\right) \\
S_{22}\left(k_{1}, x_{2}, x_{2}^{\prime}, k_{3}\right)=\frac{u_{2}\left(x_{2}\right) \hat{u}_{2}\left(x_{2}^{\prime}\right)}{4 \pi^{2}} \int_{-\infty}^{\infty} \int_{-\infty}^{\infty} R_{22}\left(r_{1}, x_{2}, x_{2}^{\prime}, r_{3}\right) \\
\exp \left[-i\left(k_{1} r_{1}+k_{3} r_{3}\right)\right] d r_{3} d r_{1} .
\end{gathered}
$$


In (2.6) $\hat{u}_{2} \equiv\left(\overline{u_{2}^{2}}\right)^{\frac{1}{2}}$ is the component turbulent intensity. The assumption of homogeneity in planes parallel to the wall allows $R_{22}$ to be a function of $r_{1}$ and $r_{3}$, but requires that the $x_{2}$ and $x_{2}^{\prime}$ correlation positions be explicitly retained.

The equation for $\phi\left(k_{1}\right)$ obtained by substituting $(2.6)$ into $(2,5)$ requires a fivedimensional integration. The physical information required is the mean shear profile $d U_{1} / d x_{2}$, the turbulence intensity distribution $u_{2}^{\prime}\left(x_{2}\right)$, and the vertical velocity correlation coefficient $R_{22}$. Functions or models based on these turbulence measurements will produce $\phi\left(k_{1}\right)$ without reference to constants or values determined from pressure fluctuation measurements.

\subsection{Mean shear}

The velocity profiles for equilibrium boundary layers are adequately described over a large region by the law of the wall plus a contribution from the law of the wake. The wake contribution is measured by the pressure-gradient parameter $\Pi$, which is constant for a given equilibrium layer. Bull $(1968,1969)$ formulated expressions for the mean shear in an equilibrium layer by dividing the boundary layer into three regions. The inner wall region is $0 \leqslant y_{*} \leqslant 33 \cdot 2$, with $y_{*} \equiv x_{2} u_{*} / \nu$, and $u_{*}=\left(\tau_{0} / \rho\right)^{\frac{1}{2}}$ (for total stress $\tau_{0}$ ) is the friction velocity:

$$
\frac{d\left(U_{1} / u_{*}\right)}{d y_{*}}=\left[1+\frac{1}{4} y_{*}+\frac{1}{2}\left(\frac{1}{4} y_{*}\right)^{2}+\frac{1}{1300}\left(\frac{1}{4} y_{*}\right)^{6}\right] \exp \left(-\frac{1}{4} y_{*}\right)
$$

The middle region includes the overlap region and the region where the wake component is large. It omits the outermost portion where the wake law does not fair smoothly into the free stream. In this region, the mean shear is simply the derivative of the wall-wake law with a slight modification in the factor $\alpha_{c}$. For $33 \cdot 2<y_{*}$ and $y / \delta<\alpha_{0}$,

$$
\frac{d\left(U_{1} / u_{*}\right)}{d(y / \delta)}=\frac{1}{\kappa}\left[\frac{\delta}{y}+\frac{\pi}{\alpha_{c}} \Pi \sin \left(\frac{\pi}{\alpha_{c}} \frac{y}{\delta}\right)\right]
$$

(von Kármán constant $\kappa=0 \cdot 41$ ). The factor $\alpha_{c}$ is given by Bull (1968) as a function of $\Pi$. The zero-pressure-gradient value is 0.837 and it increases slightly to about 0.9 as $\Pi$ increases. We used the 0.837 value for all values of $\Pi$.

In the outside region (i.e. where the wake law is incorrect), Bull proposes, for $\alpha_{c} \leqslant y / \delta \leqslant 1$,

$$
\frac{d\left(U_{1} / u_{*}\right)}{d(y / \delta)}=\frac{1}{\kappa}\left(\frac{1-y / \delta}{1-\alpha_{c}}\right)^{m-1}
$$

It is consistent with the approximation $\alpha_{c}=$ constant to take $m=1 \cdot 67$.

\subsection{Vertical velocity turbulent intensity}

The hypothesis adopted for the overlap and outer regions of the boundary layer was that the intensity would scale with the Reynolds stresses,

$$
\hat{u}_{2}^{2} / \overline{u_{1} u_{2}}=f(y / \delta) \text {. }
$$

Data from Bradshaw $(1967 a, b)$ for two equilibrium layers $I I=3 \cdot 0$ and $1 \cdot 3$, and Klebanoff's (1954) zero-pressure-gradient layer $\Pi=0 \cdot 55$, were extracted from the published graphs, and are plotted on figure 1. Bradshaw's results show no trend with $\Pi$, but they are higher than Klebanoff's zero-pressure-gradient data. 


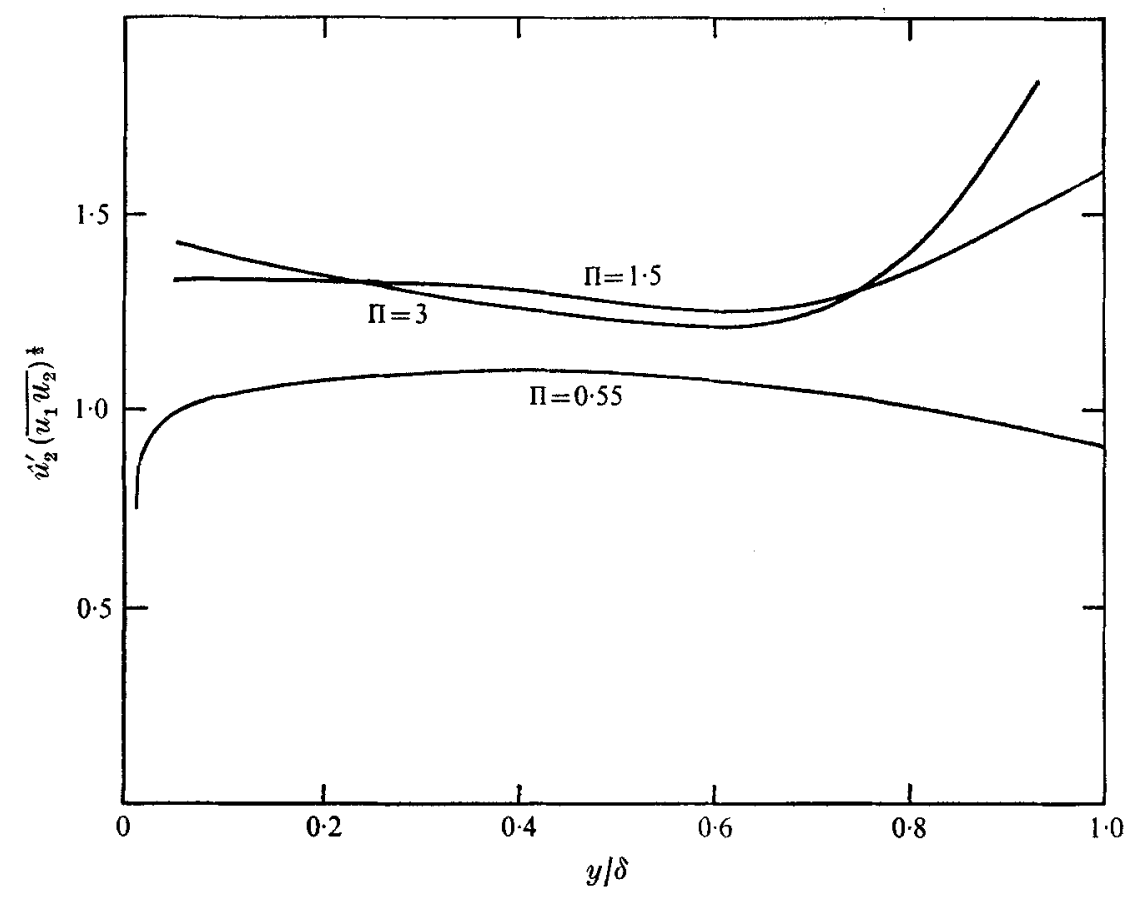

Figure 1. Influence of pressure gradient on vertical velocity turbulence intensity profiles.

Bradshaw also plots the frequency spectra of $u_{2}$, not only for adverse-pressuregradient layers, but also NPL experiments on a zero-pressure-gradient layer. These spectra are made dimensionless with the local value of $\tau / \rho$ (which is equal to the Reynolds stress in this region). Thus, the integral of the spectra is $\left(\hat{u}_{2}\right)^{2} \sqrt{u_{1} u_{2}}$. Plotting the spectra in zero pressure gradients and adverse pressure gradients together shows the curves collapse together well, and do not have a discernable trend with 11 . Therefore, we assumed that (2.10) was valid for all equilibrium layers, and not a function containing $\Pi$.

Since the intensity data should match the wall-layer data for small values of $y / \delta$, and since pressure-gradient experiments are more'difficult than zero-pressuregradient experiments, we ultimately gave more quantitative weight to Klebanoff's data, and set

$$
\left(\hat{u}_{2}\right)^{2} /\left(\overline{u_{1} u_{2}}\right)=1 \cdot 0 \text { for } y_{*}>33 \cdot 2 .
$$

This assumption converts the task into one of finding the Reynolds stress in an equilibrium boundary layer.

Mellor \& Gibson (1966), in their paper on the calculation of equilibrium velocity profiles, establish the validity of mixing length theory for these layers. The equations we adopted for calculating the total stress are

$$
\frac{\tau / \rho}{u_{*}}=\frac{l}{\delta} \frac{d\left(U_{1} / u_{*}\right)}{d(y / \delta)}
$$

with

$$
l / \delta=\kappa y / \delta \quad(0<y / \delta<0 \cdot 22) \text {, }
$$


and

$$
l / \delta=0.09 \quad(y / \delta>0 \cdot 22) .
$$

In using (2.12), the mean shear assumptions of the previous section were employed.

It was somewhat helpful in matching the outside and inside representations to make a distinction between $\tau / \rho$ and $\overline{u_{1} u_{2}}$ in the inner portion of the log region. In this region the constant stress assumption is valid, and the distribution between viscous and Reynolds stress can be found. The momentum equation with a logarithmic velocity profile simplifies to

$$
\frac{\overline{u_{1} u_{2}}}{\tau / \rho}=1-\frac{1}{\kappa y_{*}} \text {. }
$$

At the matching point of $y_{*}=33 \cdot 2, \overline{u_{1} u_{2}}$ is $93 \%$ of $\tau / \rho$. The multiplication of (2.11) $(2.12)$ and $(2.13)$ produces the final form of $\hat{u}_{2} / u_{*}$, which was used for $y_{*}>33 \cdot 2$.

In the wall layer, $y_{*}<33 \cdot 2$, the intensity data correlates in the form

$$
\widehat{u}_{2} / u_{*}=f\left(y_{*}\right)
$$

Laufer's (1954) data from a pipe were used as the basis for an algebraic expression. Actually two equations were used:

$$
\left.\begin{array}{r}
\hat{u}_{2} / u_{*}=1.20\left[0.0143 y_{*}^{2}-0.00105 y_{*}^{3}\right] \quad \text { for } \quad 0<y_{*}<8 \\
\hat{u}_{2} / u_{*}=1.20\left[0.375+0.0454 y_{*}^{\frac{1}{2}}+0.0177 y_{*}-0.000393 y_{*}^{2}\right] \\
\text { for } 8<y_{*}<33 \cdot 2
\end{array}\right\}
$$

A curve fit to Laufer's data determined the shape, then the equations were scaled upward by about $20 \%$. This was necessary to match the outer layer equation at $y_{*}=33 \cdot 2$.

In summary: for $y_{*}>33 \cdot 2$, the vertical velocity intensity was found from Bradshaw's data to have a constant relation to the Reynolds stress. The constant was chosen as unity on the basis of Klebanoff's data, and the need to match with the inner representation. The total stress was corrected for viscous contributions as $y_{*}=33.2$ was approached, and calculated using mixing length theory and Bull's velocity gradient equations. Inner-layer intensity was a curve fit to Laufer's data, boosted to make a better match with the outer region.

\subsection{Vertical velocity correlation}

The construction of $S_{22}$ (see (2.6)) was based on an isotropic turbulence model modified in two respects. The streamwise scale was allowed to be longer by a factor $\alpha$ than the scales in the other directions; and the integral scale was allowed to vary across the boundary layer. The effect on the eddies of mean straining, which gives the vorticity at $45^{\circ}$ orientation, was not included. Models of this phenomenon involve additional integration. Kraichnan (1956 b) estimated the effect of staining and of scale anisotropy on the mean-square pressure. $\mathrm{He}$ concluded that the scale influence was much greater than the orientation influence. With this in mind we decided that the added complexity of including orientation was unwarranted. 
Isotropic turbulence has a correlation coefficient of the form

$$
\begin{gathered}
R_{22}\left(r_{1}, r_{2}, r_{3}\right)=F(r)+\frac{r_{1}^{2}+r_{3}^{2}}{2 r} F^{\prime}(r), \\
r^{2}=r_{1}^{2}+r_{2}^{2}+r_{3}^{2} .
\end{gathered}
$$

The longitudinal correlation function was taken as

$$
F(r)=\exp (-r / \Lambda)
$$

(integral scale $\Lambda$ ). This form is known to fit the data slightly better than $\exp \left(-r^{2}\right)$, which is frequently used for mathematical convenience.

Scale anisotropy in the flow direction is introduced by redefining $r_{1} \rightarrow r_{1} / \alpha$. Changes across the layer require letting $r_{2}^{2}=\left(y-y^{\prime}\right)^{2}$ and $\Lambda=\Lambda\left(y, y^{\prime}\right)$. Since the integration to produce $S_{22}$ is on $r_{1}$ and $r_{3}$, it was convenient to use polar coordinates; $r^{2}=r_{1}^{2} / \alpha^{2}+r_{3}^{2}$ and $\theta=-\arcsin \left(r_{3} / r\right)$. With these redefinitions of symbols, the correlation coefficient is

$$
R_{22}\left(r, \theta, y, y^{\prime}\right)=\left\{1-r^{2}\left[r^{2}+\left(y-y^{\prime}\right)^{2}\right]^{-\frac{1}{2}}(2 \Lambda)^{-1}\right\} \exp \left\{-\left[r^{2}+\left(y-y^{\prime}\right)^{2}\right]^{\frac{1}{2}} / \Lambda\right\} .
$$

Taking into account symmetry, the polar form of the Fourier transform becomes $S_{22}\left(k_{1}, y_{2}, y_{2}^{\prime}, k_{3}: \alpha\right)=\frac{u_{2}^{\prime}(y) u_{2}^{\prime}\left(y^{\prime}\right)}{4 \pi} \int_{0}^{2 \pi} \int_{0}^{\infty} R_{22} \cos \left(\alpha k_{1} r \cos \theta\right) \cos \left(k_{3} r \sin \theta\right) \alpha r d r d \theta$.

The experimental information needed in these equations is the integral scale $\Lambda$ and the scale anisotropy factor $\alpha$.

In the wall region the turbulence should scale linearly with the distance from the wall, while in the outer region the scale is the boundary-layer thickness. Both of these requirements are included in a relation

$$
\Lambda / \delta=f(y / \delta)
$$

where $f$ is linear near the wall and constant in the outer layer. To fix $\Lambda$, we looked at Grant's (1958) data on a zero-pressure-gradient boundary layer. He published data at $y / \delta=0 \cdot 04,0 \cdot 09,0 \cdot 17$ and $0 \cdot 45$. The values of $\Lambda$ were computed by curve fitting $R_{22}\left(r_{1}, 0,0\right), R_{22}\left(0, r_{2}, 0\right)$ and $R_{22}\left(0,0, r_{3}\right)$. A relationship which fairly accurately matched the computed $\Lambda$ was linear from 0 to $y / \delta=0.22$ and constant thereafter:

$$
\left.\begin{array}{c}
\Lambda / \delta=0.63 y / \delta \text { for } 0<y / \delta<0.22, \\
\Lambda / \delta=0.14 \text { for } 0.22<y / \delta .
\end{array}\right\}
$$

As a matter of interest, the integral scale is roughly 1.5 times the mixing length scale. In view of Mellor's success with the same mixing length for all equilibrium boundary layers, and without data to indicate any substantial change, we used the expressions above for all values of $\Pi$.

The streamwise anisotropy factor $\alpha$ enters the spectral calculation for $\phi\left(k_{1}\right)$ only as a parameter. One may then take the physically reasonable viewpoint that the anisotropy changes with wavenumber $\alpha=\alpha\left(k_{1}\right)$. If this is done, then we must regard the turbulence model as being a modification of the $S_{22}$ for isotropic turbulence. Then $R_{22}$ must be computed with the $k_{1}$ integration including the $\phi\left(k_{1}\right)$ 


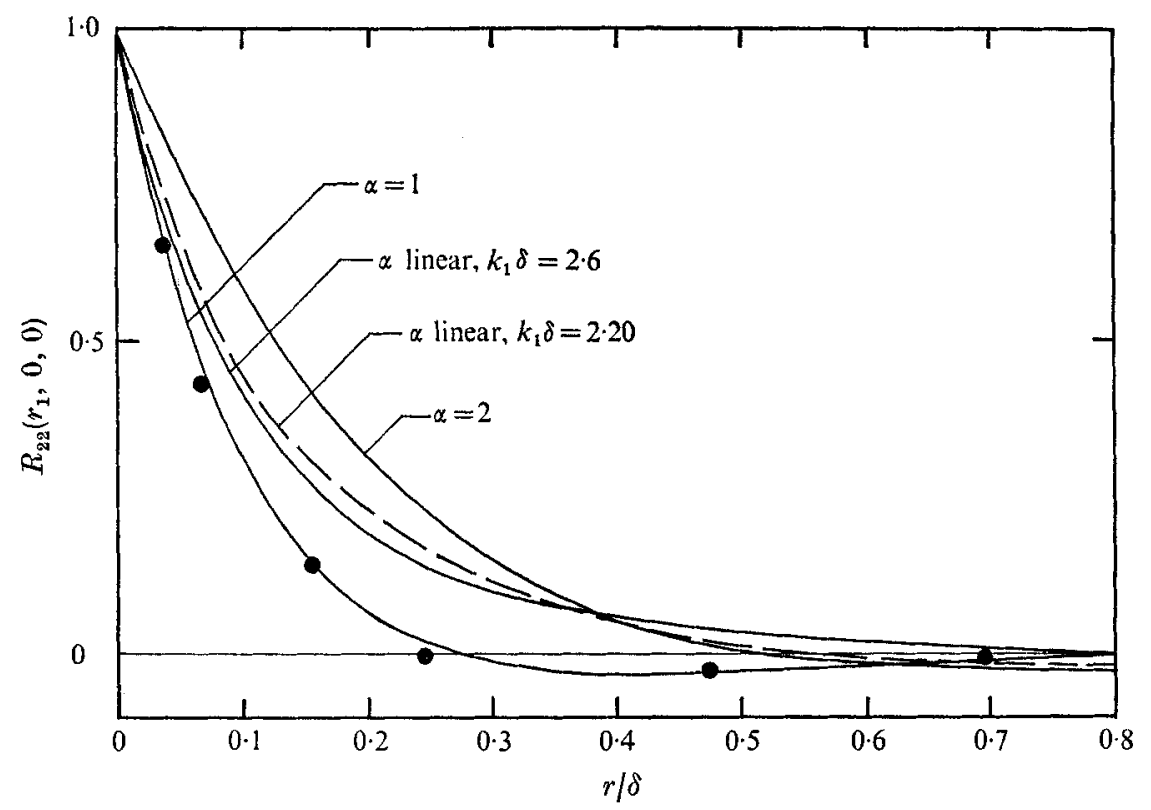

Figure 2. Correlation coefficients for anisotropic model. Data from Grant with fixed probe at $y / \delta=0 \cdot 45$.,$R_{22}\left(r_{1}, 0,0\right)$.

dependence. If $\alpha$ is constant, then it makes no difference whether one regards the model as being constructed on $R_{22}$ or $S_{22}$. The curves we present for $\phi\left(k_{1}\right)$ will be for several values of $\alpha$, and it is possible for the reader to make his own assumption for $\alpha\left(k_{1}\right)$.

An assumption for $\phi\left(k_{1}\right)$ can be tested by computing the correlation coefficients and comparing with experimental data. This is done with only one-dimensional dependence. Define the wavenumber spectrum

$$
\hat{S}_{22}\left(k_{1} \Lambda\right)=\frac{1}{2 \pi} \int_{-\infty}^{\infty} R_{22}\left(r_{1}, 0,0\right) \exp \left(-i k_{1} r_{1}\right) d\left(r_{1} \Lambda\right)
$$

With the isotropic $R_{22}$, this transform is

$$
\hat{S}_{22}\left(k_{1} \Lambda\right)=\frac{1+3\left(\alpha k_{1} \Lambda\right)^{2}}{2 \pi\left[1+\left(\alpha k_{1} \Lambda\right)^{2}\right]^{2}}
$$

The actual $R_{22}\left(r_{1}, 0,0\right)$ was computed by substituting the $\alpha\left(k_{1}\right)$ assumption, and using a numerical fast Fourier transform to inverse transform (2.22). Figure 2 shows the results of four different assumptions: $\alpha=1 ; \alpha=2 ; \alpha=2$ for $k_{1} \delta$ less than 2, decreasing linearly to $\alpha=1$ at $k_{1} \delta=6, \alpha=1$ for $k_{1} \delta$ greater than 6 ; $\alpha=2$ for $k_{1} \delta$ less than 2 , decreasing linearly to $\alpha=1$ at $k_{1} \delta=20$ and constant at one thereafter. The experimental data of Grant are shown for comparison. The broad negative loop in the data is not represented very well by any of the assumptions.

Similar calculations were made for $R_{11}\left(r_{1}, 0,0\right)$ and are plotted in figure 3 . Here the model shows a better fit with the data as $\alpha$ varies with $k_{1}$. For comparison Grant's $R_{22}\left(0, r_{2}, 0\right)$ measurements are also plotted. These data are theoretically equal to $R_{11}\left(r_{1}, 0,0\right)$ with $\alpha=1$ and a good agreement is obtained. 


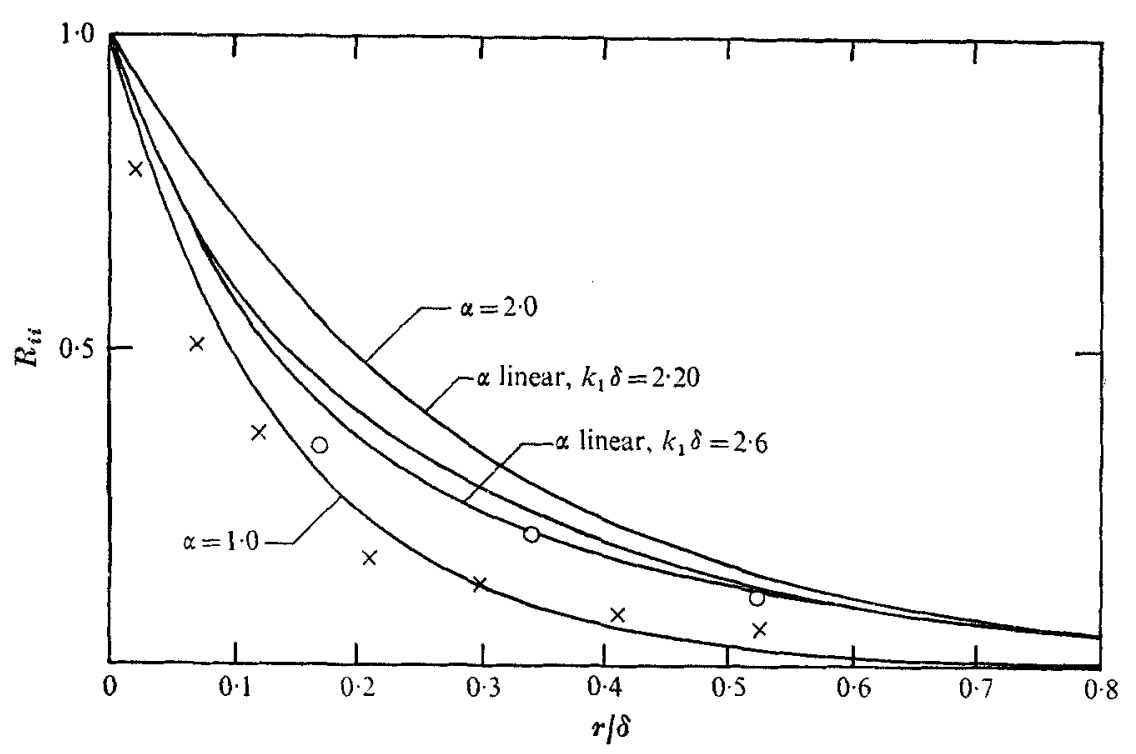

FigURE 3. Correlation coefficients for anisotropic model. Data from Grant with fixed probe at $y / \delta=0.45 . \bigcirc, R_{11}\left(r_{1}, 0,0\right) ; \times, R_{22}\left(0, r_{2}, 0\right)$.

\section{Mathematical solution}

The five-fold multiple integration to find $\phi\left(k_{1}\right)$ for each value of $k_{1}$ was done numerically. The Monte Carlo technique was employed, since the more popular quadrature techniques are uneconomical for multiple integrals of this dimension. The rate of convergence of a Monte Carlo integration theoretically depends upon the variance of the integrand, and is independent of the dimension of the integration. Success of application of this technique is in the analyst's ability to smooth out the integrand and reduce its variance by transformations and other tricks. If the integrand were made perfectly flat, only one sample would be required to find its mean value, and thus the integral.

\subsection{Monte Carlo method}

The Monte Carlo method can be viewed as a statistical process of finding the mean value of the integrand in the interval of integration. One can get a random estimate of $f_{\text {mean }}$ by evaluating $f$ at a random choice of the independent variable.

$$
I=\int_{a}^{b} f(x) d x=(a-b) f_{\text {mean }}=(a-b) \frac{1}{N} \sum_{i=1}^{N \rightarrow \infty} f\left(x_{i}\right) .
$$

Theoretically the average of a sequence of evaluations of $f$ at random $x_{i}$ 's will converge as $N \rightarrow \infty$. The error in the estimate of the integral is proportional to $\sigma / N^{\frac{1}{2}}$, where $\sigma^{2}$ is the variance of the integrand. To improve the convergence, it is helpful to transform the integration variable in such a way as to flatten $f$. Consider a function $g(x)>0$ which is normalized to integrate to 1 on $(a, b)$. One tries 
to choose $g(x)$ to have a shape similar to $f(x)$, so that $f(x) / g(x)$ has a small variance. Another important requirement is that $g(x)$ be analytically integrable.

$$
\begin{gathered}
I=\int_{a}^{b} f(x) d x=\int_{a}^{b} \frac{f(x)}{g(x)} g(x) d x=\int_{0}^{1} \frac{f(x=h(u))}{g(x=h(u))} d u \\
u=\int_{a}^{x} g\left(x^{\prime}\right) d x^{\prime}=H(x) .
\end{gathered}
$$

where

The new independent variable $u$ is a single-valued function of $x$ given by a simple expression. This expression may be inverted either analytically, or as a last resort numerically:

$$
x=h(u)
$$

With the transformed integral, the process is to choose $N$ random numbers $u_{i}$ on the interval $(0,1)$, and estimate the integral by

$$
I=\frac{1}{N} \sum_{i=1}^{N} \frac{f\left(x=h\left(u_{i}\right)\right)}{g\left(x=h\left(u_{i}\right)\right)}
$$

The judicious choice of $g(x)$ hopefully keeps $N$ to a reasonable size.

\subsection{Breakdown of integration region}

Physical and mathematical reasons motivated breaking the integrand into two parts, and splitting integrations across the boundary layer into three regions. The complete problem can be expressed by combining $(2.11),(2.18)$ and $(2.6)$ into the form

$$
\begin{aligned}
& \frac{\phi\left(k_{1}\right)}{\tau_{0}^{2} \delta}=\frac{8 \alpha k_{1}^{2}}{\pi^{2}}\left(I_{1}-I_{2}\right)=\frac{8 \alpha k_{1}^{2}}{\pi^{2}}\left\{\iiint \iint f_{1}-\iiint \iint f_{2}\right\}, \\
& I_{2}=\int_{0}^{\infty} \int_{0}^{1} \int_{0}^{1} \int_{0}^{\infty} \int_{0}^{2 \pi} \mathscr{F} r \exp \left\{-\left[r^{2}+\left(y-y^{\prime}\right)^{2}\right]^{\frac{1}{2}} / \Lambda\right\} d \theta d r d y d y^{\prime} d k_{3}, \\
& I_{2}=\int_{0}^{\infty} \int_{0}^{1} \int_{0}^{1} \int_{0}^{\infty} \int_{0}^{2 \pi} \mathscr{F} \frac{r^{3}}{2 \Lambda\left[r^{2}+\left(y-y^{\prime}\right)^{2}\right]^{\frac{1}{2}}} \\
& \times \exp \left\{-\left[r^{2}+\left(y-y^{\prime}\right)^{2}\right]^{\frac{1}{2}} / \Lambda\right\} d \theta d r d y d y^{\prime} d k_{3} \\
& \mathscr{F}\left(k_{3}, y, y^{\prime}, r, \theta\right)=\frac{1}{k^{2}} \exp \left[-k\left(y+y^{\prime}\right)\right] \frac{d U_{1}}{d y}(y) \frac{d U_{1}}{d y}\left(y^{\prime}\right) \hat{u}_{2}(y) \hat{u}_{2}\left(y^{\prime}\right) \\
& \times \cos \left(\alpha k_{1} r_{1} \cos \theta\right) \cos \left(k_{3} r \sin \theta\right) .
\end{aligned}
$$

On the right-hand side the variables have been made non-dimensional with $\delta$ and $u_{*}$ without change in notation. The left-hand side $\phi\left(k_{1}\right) / \tau_{0}^{2} \delta$ is a nondimensional spectral density, where $\tau_{0}=\rho u_{*}^{2}$ is the wall shear stress.

The boundary layer was divided into a wall region $0<y_{*}<33 \cdot 2$, a middle region $y_{*}=33 \cdot 2$ to $y / \delta=0 \cdot 2$, and an outer region $y / \delta>0 \cdot 2$. The middle region is essentially the log region and the outer region contains the wake component. 
Since there are two integrations across the layer, each $I$ integral breaks up into six distinct other integrals:

$$
I=\iiint \iint_{i n-i n}+2 \iiint \iint_{i n-m}+2 \iiint \int_{i n} \int_{o t}+\iiint \iint_{m-m}+2 \iiint \iint_{m} \int_{o t}+\iiint \iint_{o t-o t}
$$

Now the problem has expanded to require evaluation of 12 five-dimensional integrations.

\subsection{Variance reduction}

The trick required in Monte Carlo integration is in finding a variance-reducing transformation. In the final formulation, the integrals of (3.5) were written in the form

$$
\begin{aligned}
& \left.\begin{array}{rl}
I_{1} & \left.=\iiint \iint \frac{f_{1}}{g_{1}(r) g_{3}\left(k_{3}, y, y^{\prime}\right)} d \theta g(r) d r g_{3}\left(k_{3}, y, y^{\prime}\right) d y d y^{\prime} d k_{3}\right) \\
& =\iiint \iint \frac{f_{1}}{g_{1} g_{3}} d \theta d q_{1} d u d v d w, \\
I_{2} & =\iiint \iint \frac{f_{2}}{g_{2}(r) g_{3}\left(k_{3}, y, y^{\prime}\right)} d \theta d q_{2} d u d v d w,
\end{array}\right\}
\end{aligned}
$$

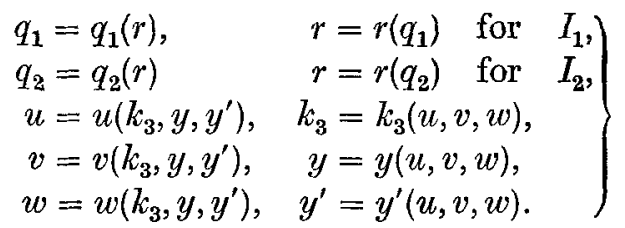

The $\theta$ variable did not require a transformation. Different transformations were required on $r$ in $I_{1}$ and $I_{2}$. The choice for $g_{1}(r)$ includes a constant $C$ which was fixed by trial numerical experiments:

$$
g_{1}(r)=C_{1}^{2} r \exp \left(-r C_{1}\right)
$$

The new variable $q$ is defined by

$$
q_{1}=\int_{0}^{r} g(r) d r=1-\left(1-r C_{1}\right) \exp \left(-r C_{1}\right) .
$$

This equation must be inverted, $r=r\left(q_{1}\right)$, by numerical means.

The choice of $g_{2}(r)$ was

yielding

$$
\left.\begin{array}{rl}
g_{2}(r) & =\frac{1}{2} C_{2}^{3} r^{2} \exp \left(-r C_{2}\right), \\
q_{2} & =1-\left(1+C_{2} r+\frac{1}{2} C_{2}^{2} r^{2}\right) \exp \left(-r C_{2}\right) .
\end{array}\right\}
$$

Again a numerical inversion was required to find $r=r\left(q_{2}\right)$.

The $y, y^{\prime}, k_{3}$ variables were intimately involved in the integrand of $I$ and were handled together. A choice of $g_{3}\left(k, y, y^{\prime}\right)$ specifies the Jacobian of

$$
(u, v, w) \rightarrow\left(k_{3}, y, y^{\prime}\right)
$$

and additional assumptions are necessary to define the transformation completely.

We chose to restrict the transformation to the form

$$
u=u\left(k_{3}\right), \quad v=v\left(k_{3}, y\right), \quad w=w\left(k_{3}, y^{\prime}\right) .
$$


Then the Jacobian

is a simple product. Let

$$
q_{3}=\frac{\partial u}{\partial k_{3}} \frac{\partial v}{\partial y} \frac{\partial w}{\partial y^{\prime}}
$$

$$
\left.\begin{array}{rl}
\frac{\partial u}{\partial k_{3}} & =\frac{2}{k \pi}, \\
\frac{\partial v}{\partial y} & =\frac{K_{a} \exp \left(-K_{a} y\right)}{\exp \left[-K_{a} a\right]-\exp \left[K_{a} b\right]}, \\
\frac{\partial w}{\partial y^{\prime}} & =\frac{K_{e} \exp \left(-K_{e} y^{\prime}\right)}{\exp \left[-K_{e} e\right]-\exp \left[K_{e} f\right]},
\end{array}\right\}
$$

where

$$
K_{a}=k+C_{a}, \quad K_{e}=k+C_{e}, \quad k^{2}=k_{1}^{2}+k_{3}^{2} .
$$

The integration region on $y$ is $(a, b)$, and that on $y^{\prime}$ is $(e, f)$. The exact transformation is found by partial integration with the arbitrary functions of $k_{3}$ set to zero:

$$
u=\int_{0}^{k_{3}} \frac{\partial u}{\partial k_{3}} d k_{3}, \quad v=\int_{0}^{y} \frac{\partial v}{\partial y} d y, \quad w=\int_{0}^{y^{\prime}} \frac{\partial w}{\partial y^{\prime}} d y^{\prime} .
$$

These equations were inverted to yield the desired transformation

$$
\left.\begin{array}{rl}
k_{3} & =\tan \left(\frac{1}{2} \pi u\right), \\
y & =\frac{-1}{K_{a}} \ln \left\{\exp \left(-K_{a} a\right)-v\left[\exp \left(-K_{a} a\right)-\exp \left(-K_{a} b\right)\right]\right\}, \\
y^{\prime} & =\frac{-1}{K_{e}} \ln \left\{\exp \left(-K_{e} e\right)-w\left[\exp \left(-K_{e} e\right)-\exp \left(-K_{e} f\right)\right]\right\} .
\end{array}\right\}
$$

It is obvious that one can substitute the first equation into the last two to obtain $y=y(u, v)$ and $y^{\prime}=y^{\prime}(u, w)$.

\subsection{Computation method and accuracy}

The six regional integrals for each $I_{1}$ and $I_{2}$ were computed simultaneously. The integration consists of averaging $N$ samples of the integrands of (3.7). Five independent random number sequences, each uniformly distributed on $(0,1)$, were generated. A sample trial is made by assigning random numbers to $\theta / 2 \pi$, $q, u, v$ and $w$. Equations (3.14) determine $k_{3}, y$ and $y^{\prime}$; and numerical inversion of (3.9) or (3.10) supplies $q$. Next, the integrands $f_{1}$ and $f_{2}$, as defined by (3.5), are evaluated according to the assumptions of $\S 2$. Evaluation of $g_{1}$ or $g_{2}$ and $g_{3}$, from equations above, allows one to form the transformed integrands $f_{1} /\left(g_{1} g_{3}\right)$ and $f_{2} /\left(g_{2} g_{3}\right)$. Repeating this process and averaging the results gives the final answer. The computation time on a CDC 6600 with $N=5000$ was about $85 \mathrm{~s}$.

The error in a Monte Carlo integration is proportional to the standard deviation of the integrand and inversely proportional to the square root of the number of samples:

$$
\text { error } \sim \sigma / N^{\frac{1}{2}} \text {. }
$$

An estimate of the standard deviation can be made by dividing the calculation into blocks and comparing the answer from each block with the final answer. 


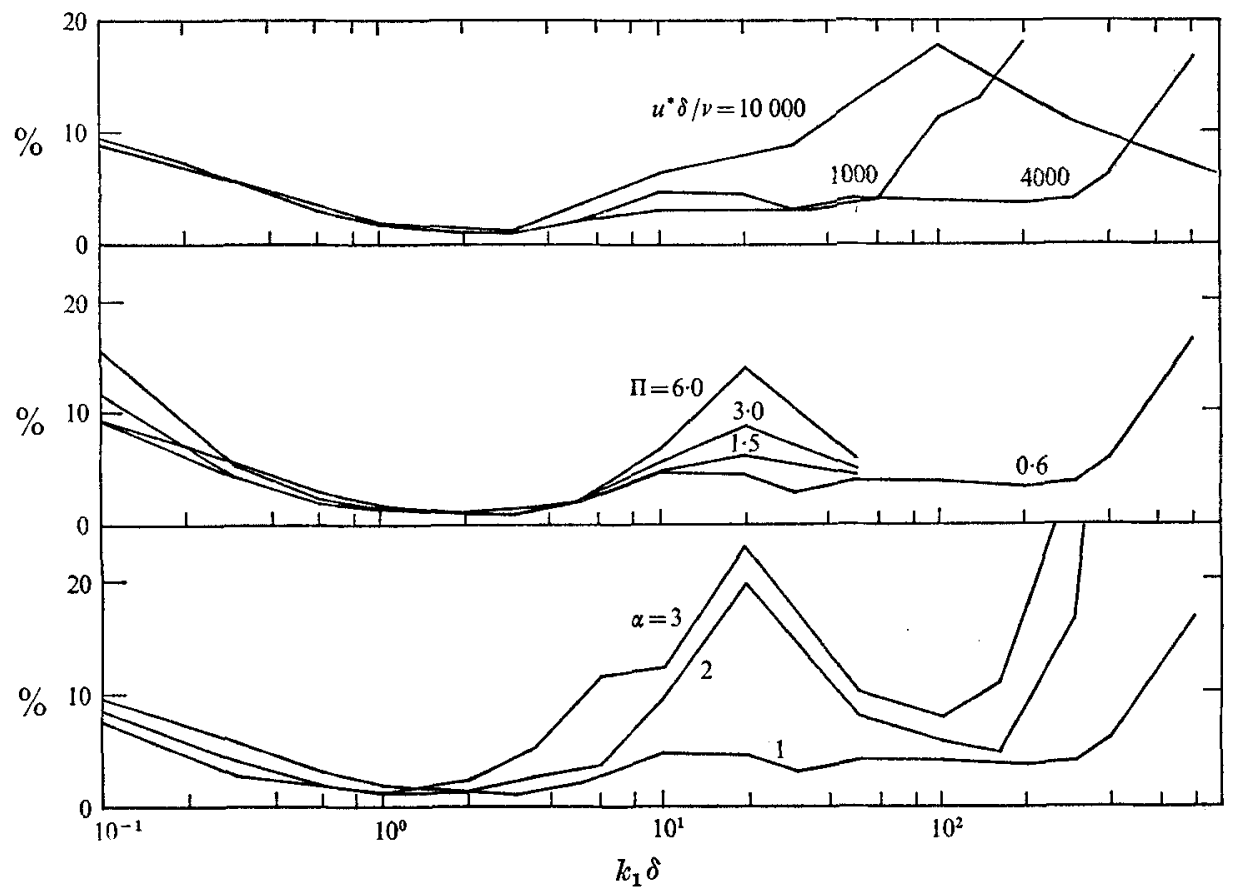

FIGURE 4. Estimated standard deviation of the integrand. $u_{*} \delta / v=1000,4000,100000$.

We used 50 blocks of 100 samples each. The answer from each block is $\phi_{100, i}$; and the final answer is $\phi_{5000}$ :

$$
\sigma=\frac{K}{50-1}\left[\sum_{i=1}^{50}\left(\phi_{100, i}-\phi_{5000}\right)^{2}\right]^{\frac{1}{2}} \text {. }
$$

The factor $K$ brings about the equality. Repeating the process for a different block size, 5000, and different computation length, 25000 , allowed us to determine that $K$ was near unity.

Figure 4 shows that the standard deviation of the integrand as a percentage of the final value. Since $N$ is the same for all of the results, this represents a relative error. The top graph shows the basic case: $u_{*} \delta / v=4000, \Pi=0 \cdot 6, \alpha=1$ ( $\nu$ kinematic viscosity). The standard deviation is less than $5 \%$ over about three decades in wavenumber. There is a tendency for $\sigma / \phi$ to increase at low wavenumbers, and to rise very rapidly at extremely high wavenumbers. When the Reynolds number is either increased or decreased, the relative error is increased at high wavenumbers. The middle graph shows the standard deviation for the different equilibrium boundary layers $\Pi=0 \cdot 6,1 \cdot 5,3 \cdot 0$ and $6 \cdot 0$. The most pronounced trend is a slight increase, and a peak in $\sigma / \phi$ at about $k_{1} \delta=20$. Later results will show that this is a transition region, where contributions from the outer portion of the boundary layer are becoming small, contributions from the middle are a maximum, and contributions from the inner layer are beginning. The bottom graph shows the effect of the anisotropy factor. The peak at $k_{1} \delta=20$ extends up to $20 \%$ for $\alpha=2$ and 3 . More significantly, the high-wavenumber 
divergence occurs sooner, and calculations showed erratic results for $k_{1} \delta>400$. Since high-wavenumber turbulence should tend toward isotropy, we did not attempt to improve the variance reduction scheme for better accuracy in this region.

\section{Results}

The wavenumber spectra $\phi\left(k_{1}\right)$ depend parametrically upon the Reynolds number $u_{*} \delta / \nu$, the pressure-gradient parameter $\Pi$, and the scale anisotropy factor $\alpha$. Calculations were made over the parameter range of physical interest, and the results are given in table 1.

\subsection{Zero-pressure-gradient spectra and Reynolds number effects}

Spectra for zero pressure gradient at three Reynolds numbers $\left(u_{*} \delta / \nu=1000\right.$, 4000 and 10000 ) are shown in figure 5. At low wavenumbers, the curves rise together with a slope of 1.1 , which increases to about 1.5 as the wavenumber increases. Previous theories, which use a constant convective velocity, have predicted that $\Phi(\omega)$ rises as $\omega^{2}=\left(k_{1} U_{c}\right)^{2}$. The current theory would require the convective velocity to decrease as $k^{-0 \cdot 3}$ to $k^{-\frac{1}{6}}$ if $\Phi(\omega)$ rises as $\omega^{2}$.

As the curves drop and come together again, they enter a region of constant -1 slope. This behaviour was not demonstrated by previous theories. This region begins at $k_{1} \delta=20$, and is an overlap region between the low-wavenumber spectra and a universal high-wavenumber spectrum. At very high wavenumbers, the curves drop away from the -1 line, in accordance with the Reynolds number. Further discussion of the high-wavenumber spectrum will be given in $\$ 4.3$.

The mean-square pressure values $\left(p^{2}\right) / \tau_{0}^{2}$ in table 1 were obtained by picking points from the curves, and using an integration routine which fits a third-order spline function to the data. The tabulated numbers are the integral values from $k_{1} \cdot \delta=0 \cdot 1$ to 100 using 40 points. The actual value of the mean-square pressure depends upon the high-wavenumber tail. In this region $\phi\left(k_{1}\right) \sim\left(k_{1} \delta\right)^{-1}$, until it drops off more steeply at about $k_{1} \nu / u=10^{-1}$. This integral is computed explicitly, and must be added to the tabulated numbers:

$$
\int_{k_{1} \delta=10^{3}}^{k_{1} \delta=10^{-1} u_{*} \delta / \nu} \phi\left(k_{1} \delta\right) / \tau_{0}^{2} \delta d\left(k_{1} \delta\right)=1 \cdot 73 \ln \left(10^{-3} u_{*} \delta / \nu\right) .
$$

If the Reynolds number is 1000 , the integral is 0,4000 yields $2 \cdot 40$ and 10000 yields $3 \cdot 98$. This represents a significant effect of Reynolds number, which is outside the range of most transducer resolution.

Spectra calculations required an integration across the boundary layer twice. Each of these integrations was broken into three parts: an inner region $y_{*}<33 \cdot 2$, a middle region roughly corresponding to the overlap region $y_{*}>33 \cdot 2$ and $y / \delta<0 \cdot 2$, and an outer region $y / \delta>0 \cdot 2$. The computational program kept track of the contributions from each regional integral; and they are plotted in figure 6 as percentages. The upper graph on the figure gives the $u_{*} \delta / \nu=1000$ case while the 10000 case is on the lower half. Figure 7 contains the $u_{*} \delta / \nu=4000$ case as part of another comparison. 


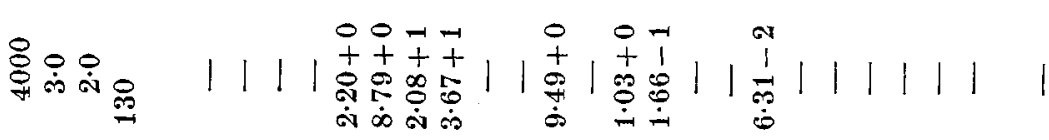

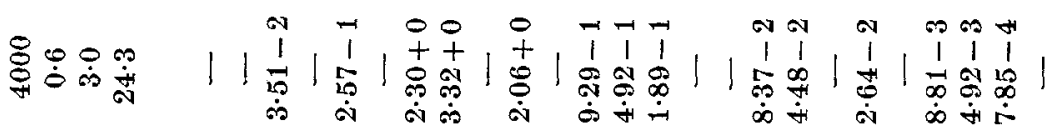

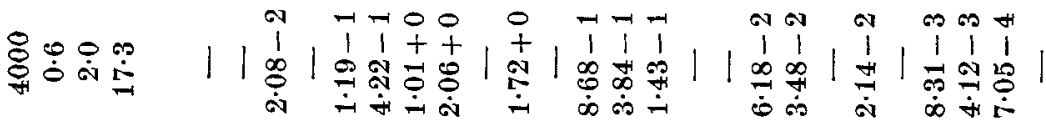

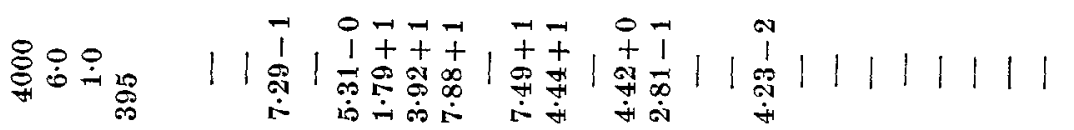

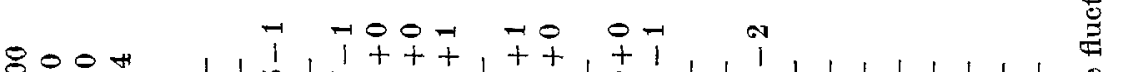

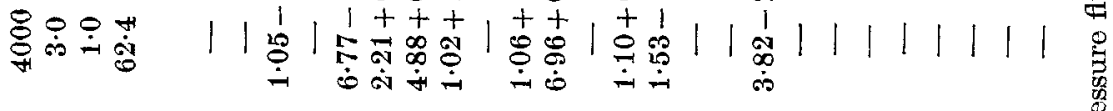

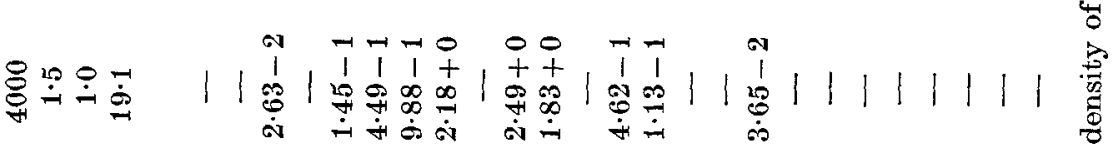

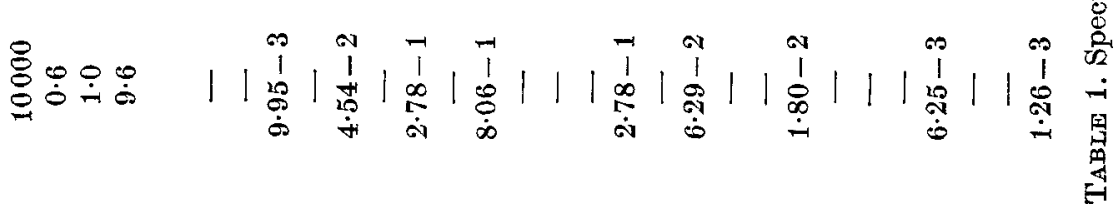

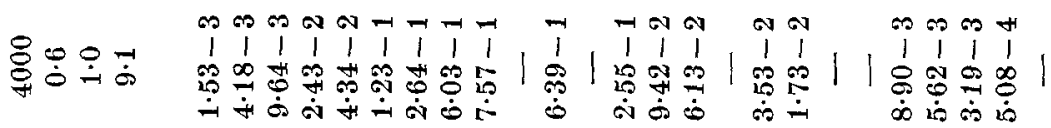

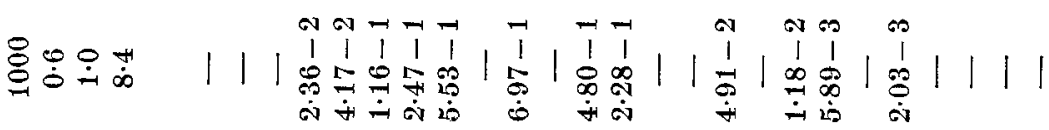

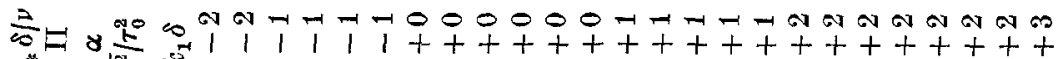

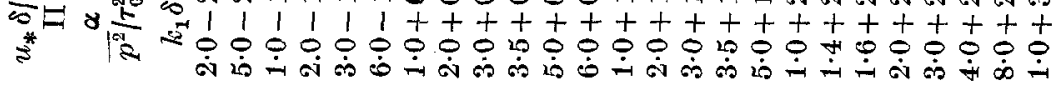




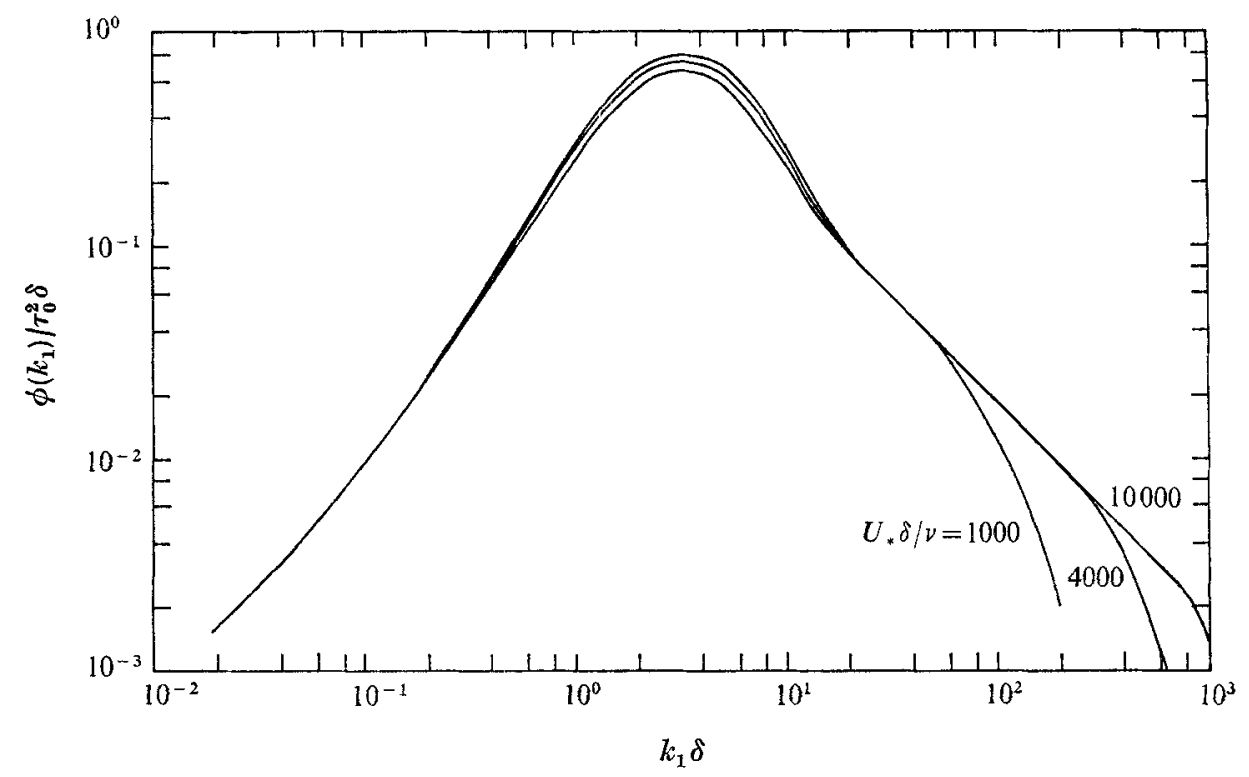

Figure 5. Wavenumber spectra in zero pressure gradient. $\Pi=0 \cdot 6, \alpha=1$.

Several things are noticeable. All outer-region contributions become nil at about $k_{1} \delta=20$, irrespective of Reynolds number. This is the point where the -1 power region begins in the spectra. The major influence of a lower Reynolds number is to cause the inner region contributions to pick up at a lower wavenumber. This pinches the middle region contributions into a smaller wavenumber band. The inner-inner contribution dominates the spectrum at the highest wavenumbers. When this contribution is about $90 \%$, the spectrum begins rapidly to drop below the -1 line. One unexpected result is that the outer-region contributions reach a peak, and the middle region becomes dominant again at the very lowest wavenumbers. This is most clearly shown in figure 9 . These figures clearly show the non-local factors that determine the pressure fluctuations.

Experimental data are frequently normalized using dynamic pressure $q$. The organization and assumptions of our theory imply that $\phi\left(k_{1}\right) / q^{2} \delta$ has considerably more variation with Reynolds number than $\phi\left(k_{1}\right) / \tau_{0}^{2} \delta$. The -1 slope region would also occur at a different level when $q$ is used as a factor.

\subsection{Anisotropy and turbulence scale effects}

The scale anisotropy factor $\alpha$ is the ratio of the integral scale in the flow direction to that in the transverse direction. $\alpha$ enters the calculation for each $\phi\left(k_{1}\right)$ point only as a parameter. Therefore it is possible to consider $\alpha\left(k_{1}\right)$, and connect together points with the proper $\alpha$ into a final spectrum. It is only for convenience that we have connected together curves where $\alpha$ is constant. Figure 7 displays curves with $\alpha=1,2$ and 3, for a zero-pressure-gradient layer at $u_{*} \delta / \nu=4000$. The corresponding regional contributions are plotted in figure 8 for $\alpha=1$ and 3 . Trends in the regional contributions for $\alpha=2$ are intermediate between these cases.

In general, anisotropy increases the level of the spectrum and moves the entire curve to lower wavenumbers. There is little change in shape. Overlapping the 
Wall pressure spectra calculations

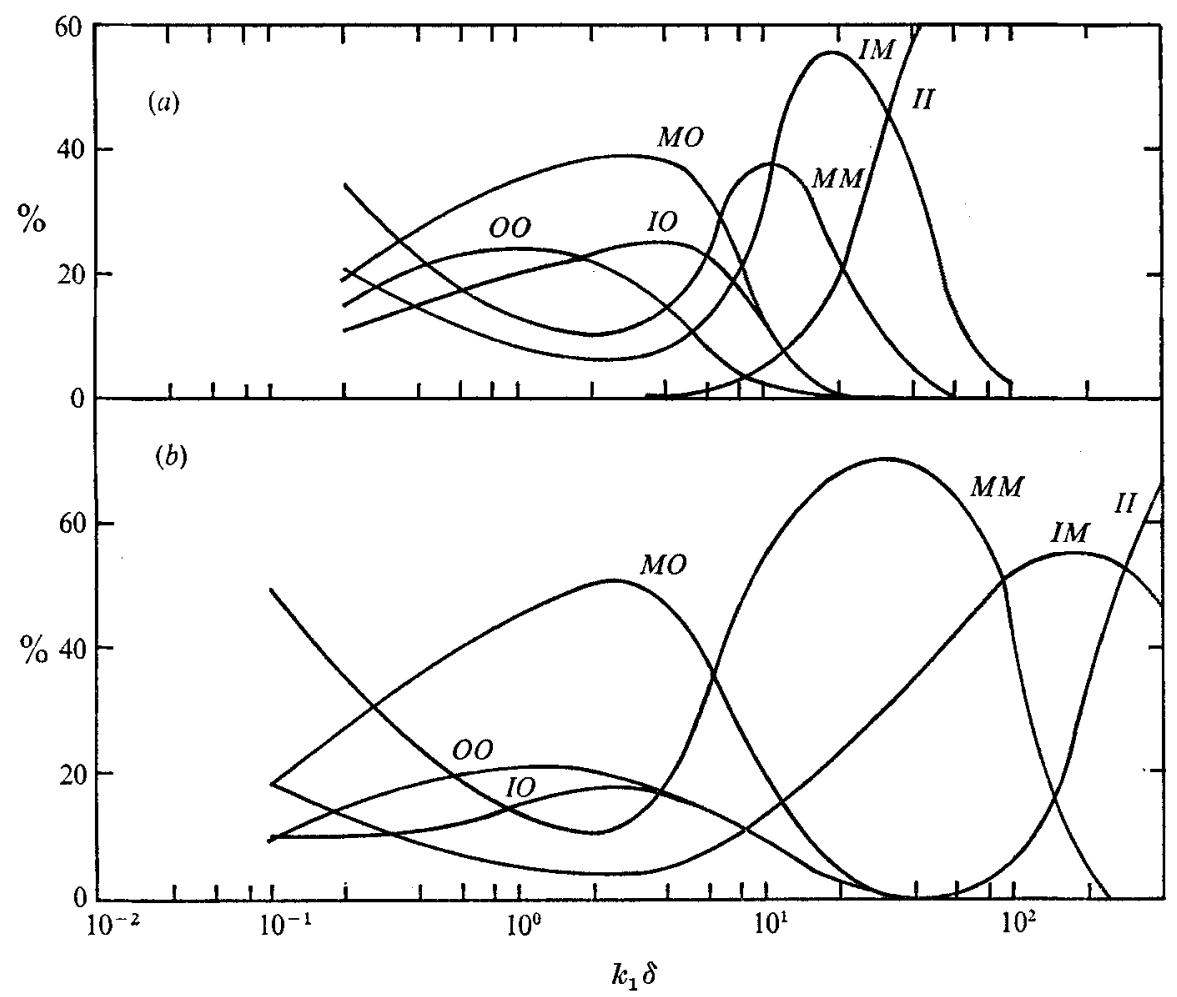

FIGURE 6. Regional contributions in zero pressure gradient. $I=$ inner region $0 \leqslant y_{*} \leqslant 33 \cdot 2$; $M=$ middle region $33 \cdot 2<y_{*}, y / \delta<0 \cdot 2 ; O=$ outer region $y / \delta>0 \cdot 2 . \Pi=0 \cdot 6, \alpha=1$. (a) $u_{*} \delta / \nu=1000,(b) u_{*} \delta / \nu=10000$.

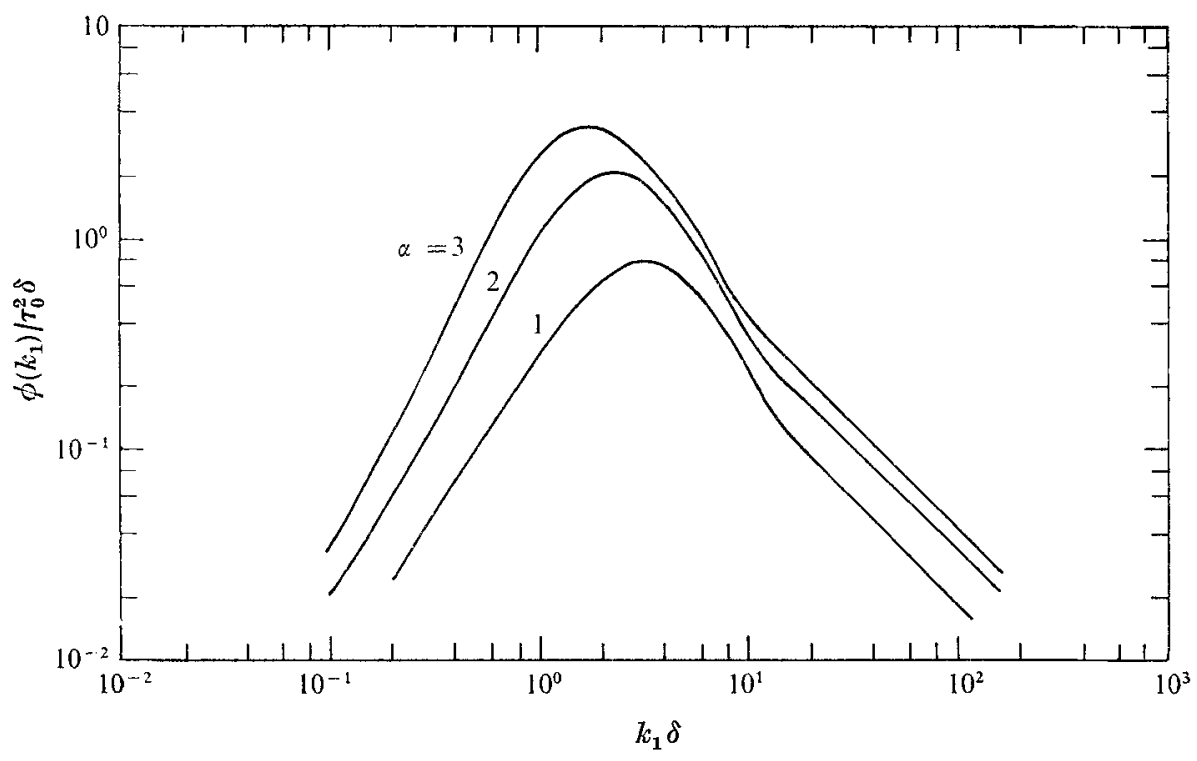

Figure 7. Effects of anisotropy on zero-pressure-gradient spectra. $\Pi=0 \cdot 6, u_{*} \delta / \nu=4000$. 


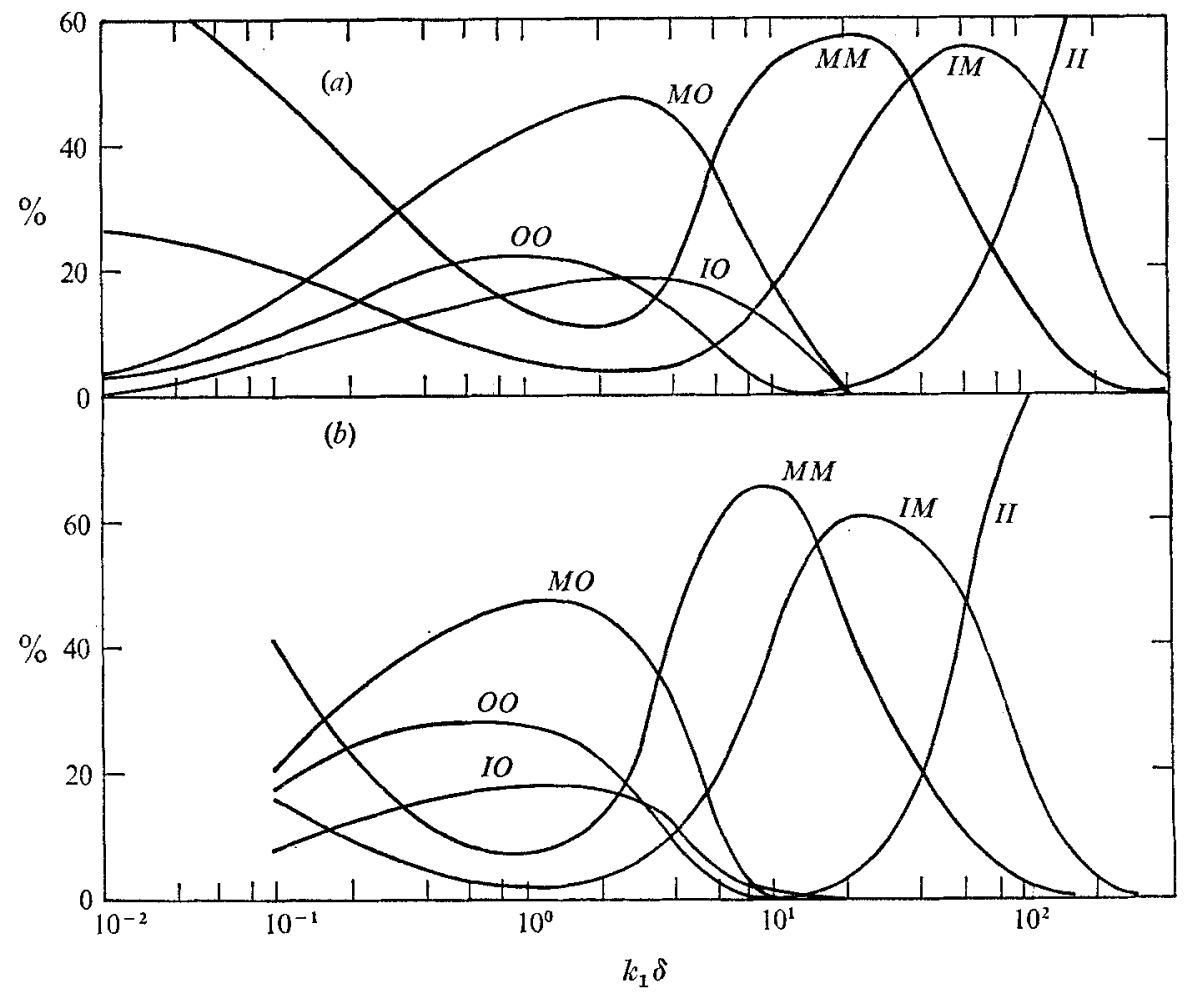

FIGURE 8. Effects of anisotropy on regional contributions. $\Pi=0 \cdot 6, u_{*} \delta / \nu=4000$. (a) $\alpha=1,(b) \alpha=3$.

curves shows a slightly sharper peak for higher $\alpha$. The peak value of $\phi\left(k_{1}\right)$ increases by $300 \%$ as $\alpha$ changes from 1 to 2 . The regional contributions also show the same general character with a shift to lower wavenumbers.

During the development of the program we found that the integral scale was influential in fixing the level of the curves. The sensitivity of this assumption was tested by inserting two constants $C_{\lambda}, B_{\lambda}$ into the integral scale function.

$$
\frac{\Lambda}{\delta}=C_{\lambda} f\left(B_{\lambda} \frac{y}{\delta}\right)
$$

Calculations for three different wavenumbers gave a $\pm 30 \%$ change in $\phi\left(k_{1}\right)$ for $a \pm 10 \%$ change in $C_{\lambda}$. The sensitivity to $B_{\lambda}$ was not as large; $\pm 15 \%$ change in $\phi\left(k_{1}\right)$ for $\pm 10 \%$ change in $B_{\lambda}$.

\subsection{High-wavenumber spectrum and overlap region}

When contributions to the spectra come entirely from the wall layer and the $\log$ region, it is possible to rewrite the problem in terms of wall variables alone. The spectrum is then universal in the sense that it is independent of Reynolds number. The proper non-dimensional form is

$$
\frac{\phi\left(k_{1}\right) u_{*}}{\tau_{0}^{2} \nu}=f\left(\frac{k_{1} \nu}{u_{*}}\right)
$$




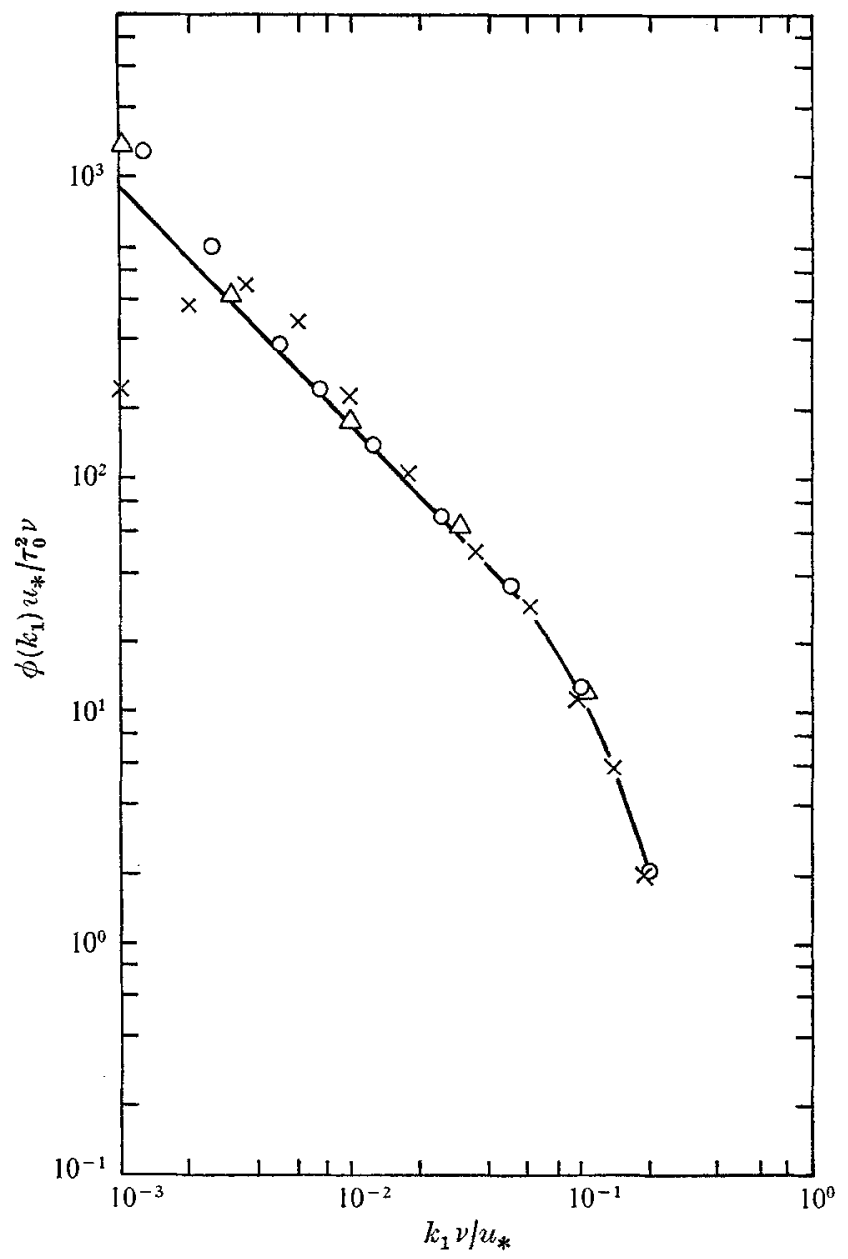

FigURE 9. High-wavenumber spectrum. Symbols are points calculated at different Reynolds numbers. $u_{*} \delta / \nu: \times, 1000 ; \bigcirc, 4000 ; \triangle, 10000$.

Contributions from the outer region become zero at $k_{1} \delta \simeq 20$, so one expects the high-wavenumber form to apply beyond this value.

The existence of an overlap region in the pressure spectrum has been a matter of speculation. Since pressure fluctuations represent the integrated effect of events across the layer, it was thought that distant points might always influence the spectrum and preclude an overlap region. Bradshaw (1967) was first to point out that an overlap region would have a -1 slope. He proposed that the region existed between $k_{1} \delta=4$ and a value of $k_{1} v / u_{*}$ inversely proportional to the sublayer thickness. He also reasoned that the mean-square pressure should depend upon Reynolds number as in (4.1).

It is interesting to review the assumptions required to find the form of $\phi\left(k_{1}\right)$ in. the overlap region. $P$ will denote the spectrum in outer variables, and $\mathscr{P}$ the spectrum in inner variables.

$$
P\left(k_{1} \delta ; \Pi\right)=\phi\left(k_{1}\right) / \tau_{0}^{2} \delta, \quad \mathscr{P}\left(k \nu / u_{*}\right)=\phi\left(k_{1}\right) u_{*} / \tau_{0}^{2} \nu, \quad R e=u_{*} \delta / \nu .
$$


There is an intermediate wavenumber where these expressions are equal; $P=\mathscr{P} / R e$. If, in addition, it turns out that the expressions are equal over a range of wavenumbers, then the equality may be differentiated:

$$
\left(k_{1} \delta\right)^{2} P^{\prime}\left(k_{1} \delta ; \Pi\right)=\left(k_{1} \nu / u_{*}\right)^{2} \mathscr{P}^{\prime}\left(k_{1} \nu / u_{*}\right)=\text { const. }
$$

For this expression to be true, $P^{\prime}$ must be independent of $I$ in the overlap region, and the spectra have the form

$$
P\left(k_{1} \delta\right)=A\left(k_{1} \delta\right)^{-1}, \quad \mathscr{P}\left(k_{1} v / u_{*}\right)=A\left(k_{1} v / u_{*}\right)^{-1}
$$

This gives a straight line of -1 slope on a log-log graph.

Our calculations produced an overlap region beginning about $k_{1} \delta=20$ and ending about $k_{1} v / u_{*}=0.5$. The high-wavenumber spectrum is shown on figure 9 . We did not redo the computer program in inner variables, but simply converted our previous results for all three Reynolds numbers. The calculated points are plotted; and the scatter indicates the consistency of the calculation procedure. The drop off below the -1 region begins once the inner regional contribution, $y_{*}<33 \cdot 2$, becomes the dominant source $(90 \%)$. Figure 7 displays the highwavenumber spectra for different anisotropy factors. These calculations could not be extended into the drop-off region, because of large relative errors. The trend appeared to be that the drop off occurs sooner for $\alpha=2$ and 3 . This is not very important, since isotropy probably prevails at these small wavelengths.

\subsection{Equilibrium pressure-gradient spectra}

Spectra for equilibrium pressure gradients $\Pi=0 \cdot 6,1 \cdot 5,3 \cdot 0$ and $6 \cdot 0$ are presented in figure 10. At low wavenumbers, the curves rise with similar slopes and peak at about the same wavenumber. There is a slight tendency for the peak to move to lower wavenumbers. The peak values increase by a factor of 100 from $\Pi=0 \cdot 6$ to $\Pi=6 \cdot 0$. On the high-wavenumber side, the curves drop steeply into the overlap region. The overlap region begins at progressively higher values of $k_{1} \delta$ as $\mathrm{II}$ increases.

Regional contributions displayed in figure 11 show a marked increase from the outer-outer and outer-middle integrals. However, these contributions always fall to zero around $k_{1} \delta=20$. Integrals over the middle and inner regions peak and fall at about the same wavenumber, irrespective of $\Pi$. It is well known that increasing the wake component decreases the upper extent of the velocity profile logarithmic region. The same effect occurs in the pressure spectrum overlap region. This is not adequately depicted in our regional contribution graphs, since the boundary of the outer and middle regions is fixed at $y / \delta=0 \cdot 2$. At higher values of $\Pi$ a significant wake component exists inside $y / \delta=0 \cdot 2$, and is therefore counted as a middle-region contribution.

\subsection{Convective velocity}

Conversion of the wavenumber spectrum $\phi\left(k_{1}\right)$ into a frequency spectrum $\Phi(\omega)$ requires knowledge of the convective velocity $U_{c}\left(k_{1}\right)$ as a function of wavenumber. In this section, the classic arguments for an overlap region will be used to derive an equation for the convective velocity. The postulate of an overlap region was 


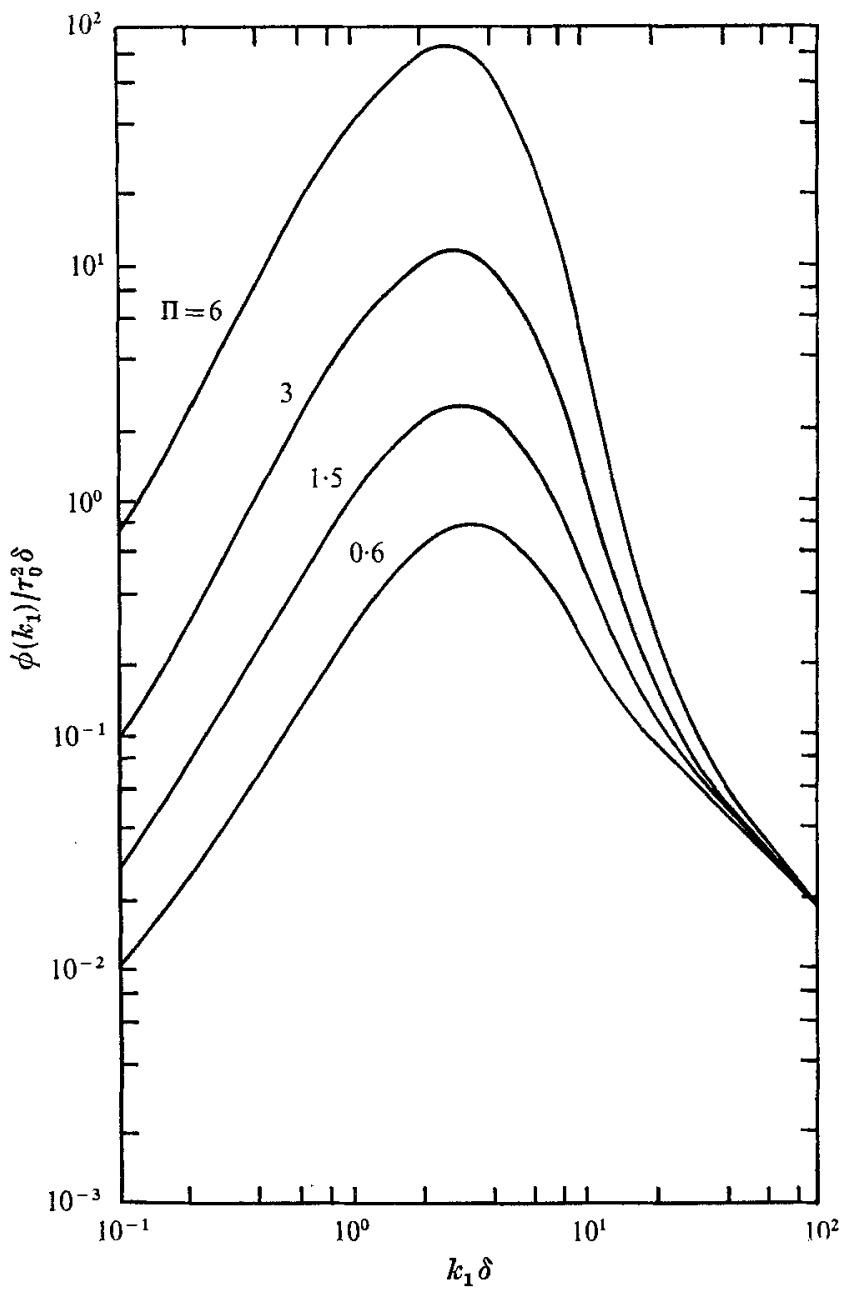

FIGURE 10. Wavenumber spectra for different pressure gradients.

$$
u_{*} \delta / v=4000, \alpha=1 \text {. }
$$

motivated by the fact that the spectra calculations displayed such a region, even though $\phi\left(k_{1}\right)$ is an integrated effect.

At low wavenumbers, the apparent origin of the pressure fluctuations is in the wake region. The convective velocity should be related in some way to the mean velocity in this region. The appropriate non-dimensional form is

$$
\left(U_{c}-U_{1}\right) / u_{*}=F\left(k_{1} \delta ; \Pi\right) \text {. }
$$

In the high-wavenumber regions, one expects scaling with the wall variables:

$$
U_{c} / u_{*}=f\left(k_{1} v / u_{*}\right) \text {. }
$$

By postulating that there exists an overlap region, one may proceed to equate and differentiate, as in $\$ 4.4$. This leads to the overlap laws

$$
\begin{aligned}
& F=\beta \ln \left(k_{1} \delta\right)+A_{1}(\Pi), \\
& f=\beta \ln \left(k_{1} \nu / u_{*}\right)+A_{2},
\end{aligned}
$$




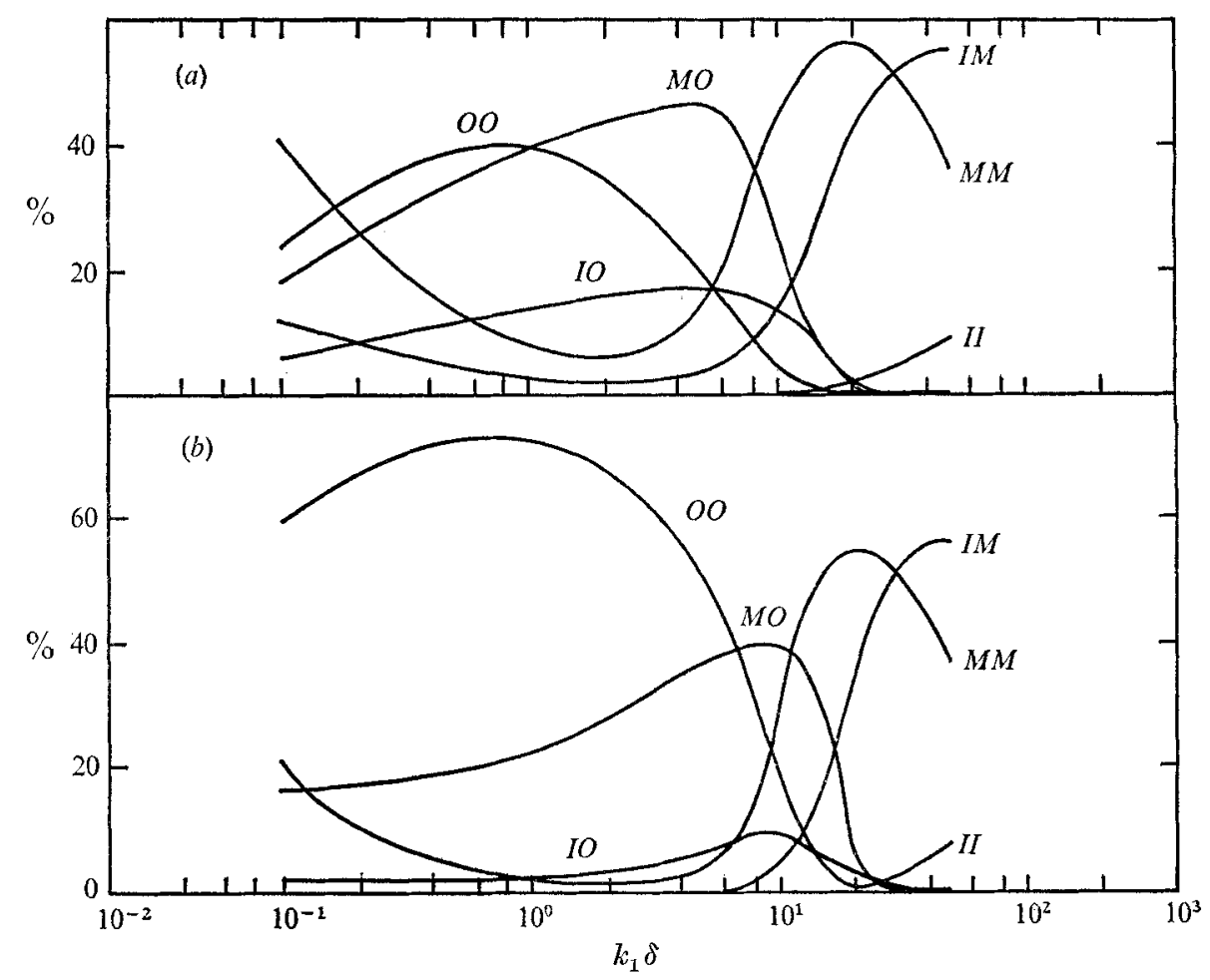

Frgure 11. Effects of pressure gradient on regional contributions.

$$
I I=0 \cdot 6, u_{*} \delta / \nu=4000, \alpha=1 \text {. }
$$

where $\beta, A_{1}$, and $A_{2}$ are coefficients. Equating the expressions again yields the friction law

$$
U_{1} / u_{*}=-\beta \ln \left(u_{*} \delta / v\right)+A_{2}-A_{1}(\Pi) .
$$

A similar friction law may be obtained from the overlap mean velocity expression. Comparison relates the coefficients to those in the mean velocity profile $(\beta=1 / \kappa$, $\left.A_{1}=-2 \Pi / \kappa, A_{2}=5 \cdot 0\right)$. Thus, not only is the convective velocity overlap law established, but the coefficients are also fixed. A slightly different form of $(4.10)$ is

$$
\frac{U_{c}}{u_{*}}=-\frac{1}{\kappa} \ln \left(k_{1} \delta\right)+\frac{1}{\kappa} \ln \frac{u_{*} \delta}{\nu}+A_{2} .
$$

This equation is compared with experimental data in figure 12.

Experimental data on $U_{c}\left(k_{1}\right)$ are given by Wills (1970) for a zero-pressuregradient layer at a Reynolds number $u_{*} \delta / \nu=2500$. His values agree with Landahl's (1967) wave theory at high wavenumbers, but are higher than the theory at low wavenumbers. Wills attributes the discrepancy between his results and those of Willmarth \& Wooldridge (1962) to differences in definitions. The convection velocity overlap law is in good agreement with Wills' data beginning around $k_{1} \delta=10$. Wills felt the data approached a constant value at high wavenumbers. The approach to a constant value at $k_{1} \delta=40$ does not agree with theory; but it would not be difficult to reinterpret the data so that the agreement would be good (see Wills, figure 11). 


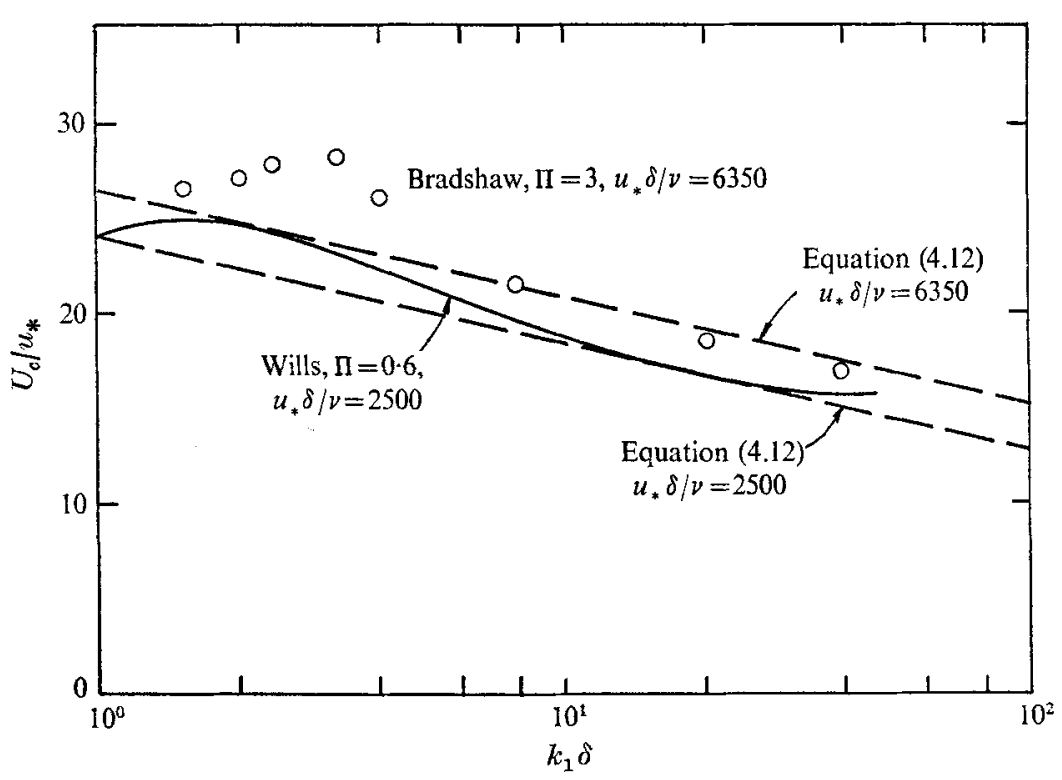

Figure 12. Comparison of overlap law with experimental data.

Bradshaw (1967) published convective velocity results for an equilibrium pressure gradient $\Pi=3 \cdot 0$ and a larger Reynolds number $u_{*} \delta / \nu=6350$. The agreement with the overlap law is very good in this case also.

At this point, the natural thing is to follow boundary-layer history, and propose a convective velocity 'wake' law. This law extends the overlap law into the low-wavenumber region, and accounts for pressure-gradient effects. The complete equation is

$$
\left.\begin{array}{rl}
\frac{U_{c}}{u_{*}} & =f\left(k_{1} \delta ; u_{*} \delta / \nu\right)+W\left(k_{1} \delta ; \Pi\right), \\
W & =\frac{2}{K} \Pi^{\frac{1}{2}}\left(k_{1} \delta-1\right) \exp \left[\frac{2}{3}\left(k_{1} \delta-1\right)\right] \quad \text { for } \quad k_{1} \delta \leqslant 1 .
\end{array}\right\}
$$

The 'wake' function $W$ has an arbitrary form. We chose the form above as a rough but reasonable fit to the data. It peaks at $k_{1} \delta=2 \cdot 5$. Including $\Pi$ in the exponential would cause the peak to shift as the data seem to suggest. The equation is proposed only for $k_{1} \delta<1$. Behaviour at lower wavenumbers is uncertain.

Convective velocity experiments are sometimes expressed as $U_{c} / U_{1}\left(\omega \delta / U_{1}\right)$ (see Wills 1970, figure 12). Assuming a skin-friction law and Taylor's frozen-flow hypothesis, one can convert (4.13) into this form. This expression should be compared to measurements with zero transducer separation, since the convection hypothesis does not account for the fact that large eddies are coherent for longer distances than small eddies. If one converts (4.13) into the frequency form, then part of the discrepancy between Wills and Willmarth's data (Wills, figure 12) is seen to be a Reynolds number effect. This also produces a Reynolds number trend exactly opposite to Landahl's. 


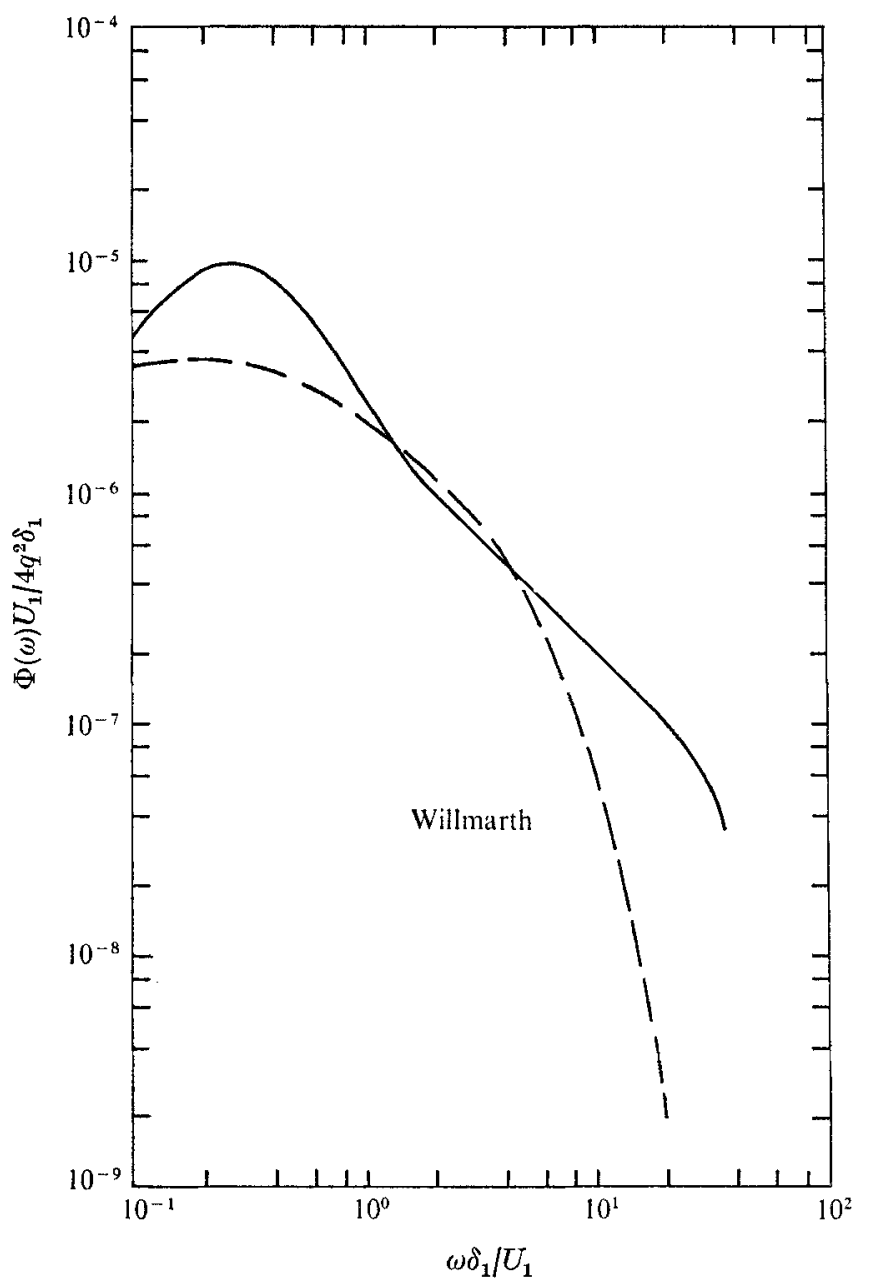

Figure 13. Frequency spectrum for zero pressure gradient compared with experimental data.

A final comment concerns the experimental tendency for $U_{c}$ to decrease slightly at very low wavenumbers. There has been some thought that the drop off was caused by extraneous factors. Some support for believing the drop off is real is given by the fact that, at low wavenumbers, the regional contributions from the outer region drop off, and the middle region contributions pick up. This begins to occur below $k_{1} \delta=1$ in our calculations. In Bradshaw's data the turnaround point is $k_{1} \delta=3$, and in Wills' it is $k_{1} \delta=1.5$.

\subsection{Frequency spectra and experimental comparison}

Frequency spectra are obtained by using the relations

$$
\Phi(\omega)=U_{c}^{-1} \phi\left(k_{1} \rightarrow \omega / U_{c}\right), \quad \omega=k_{1} U_{c}\left(k_{1}\right) .
$$

First of all we note that in the overlap region $\phi\left(k_{1}\right) \sim k_{1}^{-1}$, so (4.14) shows $\Phi(\omega) \sim \omega^{-1}$, irrespective of any equation for $U_{c}\left(k_{1}\right)$. The $k_{1}^{-1}$ line is the only line 


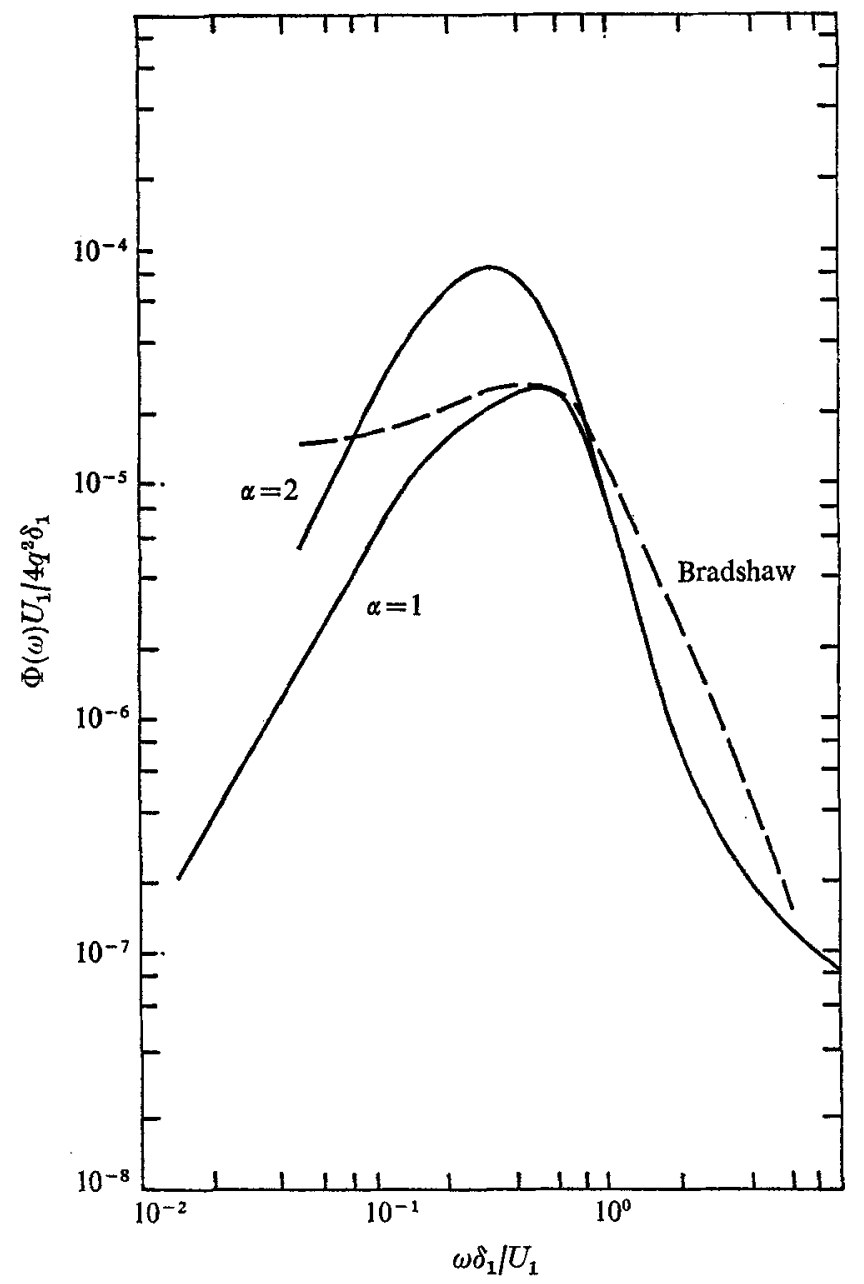

FIGURE 14. Frequency spectrum for pressure gradient $\Pi=3$ compared with experimental data.

that transforms onto the frequency graph, without change in slope (unless $U_{c}$ is constant, of course).

Experimental evidence of the overlap region will have a -1 slope on a frequency plot. Most experiments drop off faster than this rate. However, transducer resolution and correction methods are a problem at these frequencies. Wills (1970, figure 17) plots some unpublished work by Hodgson, using a 0.005 diameter 'probe microphone'. This work was also mentioned by Bradshaw (1967). It is unfortunate that the details of the experiment are unavailable. Nevertheless, the slope of these data is a little less than one, about $-0 \cdot 8$. The region is quite long; it is established at $\omega \delta_{1} / U_{1}=1$, and continues for a decade. The exact level of the data is not given, since the spectrum was normalized. For this reason it is not plotted in figure 13. Assuming the veracity of these measurements, we conclude, as did Bradshaw, that the overlap region exists, but is beyond reach of most measurements. 


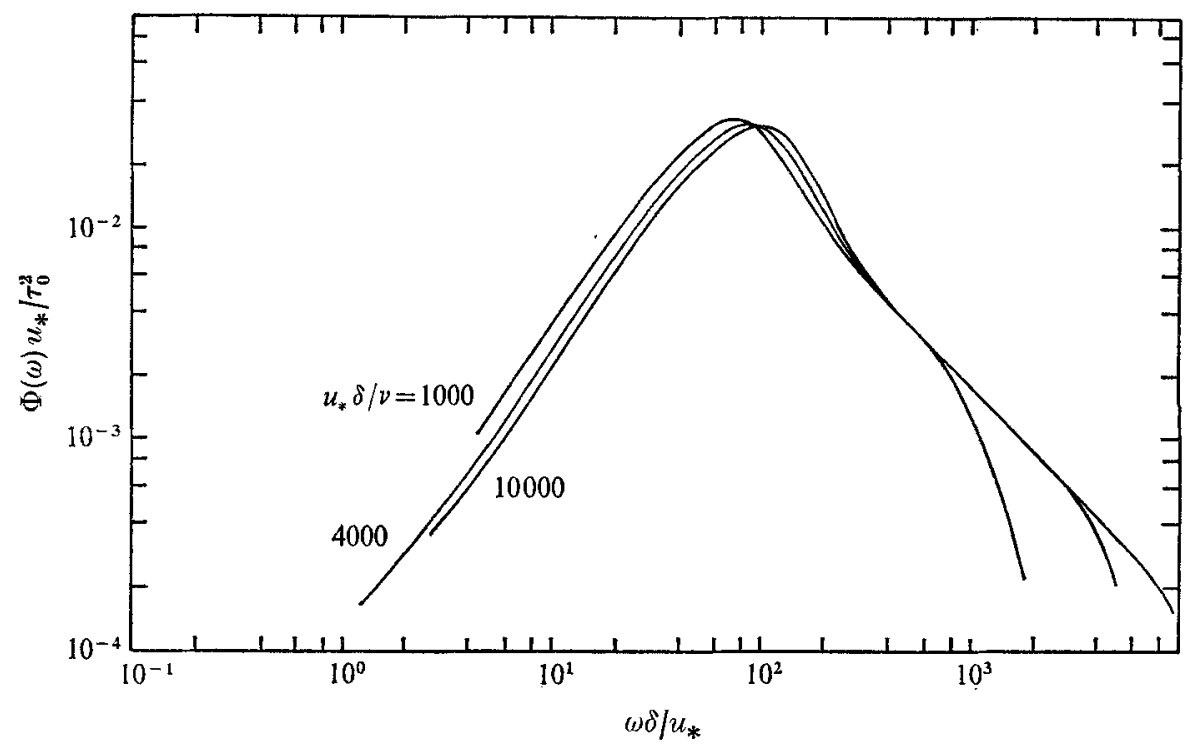

FIGURE 15. Frequency spectra normalized with $\delta$ and $u_{*} . \Pi=0 \cdot 6, \alpha=1$.

Figure 13 compares the data of Willmarth \& Roos (1965) for a zero pressure gradient with calculated curves. These data are typical of a large number of papers which agree to about a factor of 5 . Willmarth's data are the result of extrapolating measurements with microphones of different sizes. The data do not peak as strongly, nor at quite as high a level as the calculation. The rapid fall at high frequencies could possibly be attributed to breakdown in validity of the extrapolation procedure.

Data taken by Bradshaw (1967) on an equilibrium boundary layer with $\Pi=3$ are presented on figure 14 . The peak of the pressure gradient data and the calculations are in very good agreement. However, the calculations show a more rapid fall off than the experiments at high frequencies. One might also note that the overlap region is just beginning at the frequency where valid spectrum measurements end. Bradshaw attributed the slow decline in the spectrum at low frequencies to acoustic noise within the tunnel. The curve for an anisotropy factor $\alpha=2$ is also plotted. Extraneous sound is certainly important in the experiments; however, one can also attribute a portion of this spectrum to anisotropy of the large eddies.

Probably one of the best ways to close the gap between a wavenumber spectrum and an experimental frequency spectrum is to plot the data in the form $\Phi(\omega) u_{*} / \tau_{0}^{2} \delta$ against $\omega \delta / u_{*}$. These co-ordinates have been used in figure 15 , where the curves for three different Reynolds numbers are given. The parameters $u_{*}, \delta$ and $\tau_{0}=\rho u_{*}^{2}$ may be found by fitting the wall-wake equation to the velocity profile. This procedure was standardized for the AFOSR-IFP-Standford Symposium (see Coles \& Hirst 1968). Figure 15 was produced by assuming the convection velocity is given by (4.13) when $k_{1} \delta \geqslant 1$ and is constant for $k_{1} \delta \leqslant 1$. The advantage of figure 15 is that the effects of Reynolds number are minimized. 
The authors would like to thank the Aeronautical Structures Branch of NASA's Ames Research Center for support under grants NGR 37-002-083 to Oklahoma State University and NGR 44-012-221 to the University of Texas. The authors, and especially JHL, would like to express appreciation to Professor John Chandler of Oklahoma State University for advice concerning the Monte Carlo method.

\section{REFERENCES}

Batchelor, G. K. 1951 Proc. Camb. Phil. Soc. 47, 359.

BRAdSHaw, P. 1967 a J. Fluid Mech. 29, 625.

Bradshat, P. $1967 b$ J. Fluid Mech. 30, 241.

BULL, M. K. $1968 a$ A.I.A.A. J. 7, 359.

BULL, M. K. 1969 Aero. J. Royal Aero. Soc. 73, 143.

Coles, D. E. \& HrRst, E. A. 1968 Proc. Conf. on Computation of Turbulent Boundary Layers, Mechanical Engineering Department, Stanford University, vol. 2.

Corcos, G. M. 1964 J. Fluid Mech. 18, 353.

Grant, H. L. 1958 J. Fluid Mech. 4, 149.

Heisenberg, W. 1948 Z. Phys. 124, 628.

Hodgson, T.H. 1962 Ph.D. thesis, University of London.

Klebanoff, P. S. 1954 N.A.C.A.Tech. Note, no. 3178.

Kratchnav, R. H. $1956 a$ J. Acoust. Soc. Am. 28, 64.

Kraichnan, R. H. $1956 b$ J. Acoust. Soc. Am. 28, 378.

LANDAHL, M. T. 1967 J. Fluid Mech. 29, 441.

LaUfer, J. 1954 N.A.C.A. Rep. no. 1174.

LILLey, G. M. $1963 A G A R D$ Rep. no. 454.

Lildey, G. M. 1964 Arch. Mech. Stos. 2, 16.

LiLLey, G. M. \& Hodgson, T. H. 1960 AGARD Rep. no. 276.

MelLor, G. L. \& GIBson, D. M. 1966 J. Fluid Mech. 24, 225.

Panton, R. L., Lowery, R. L. \& Reischuman, M. M. 1971 Ames Research Center Rep. NASA NGR 37-002-083.

Townsend, A. A. 1956 The Structure of Turbulent Shear Flow. Cambridge University Press.

WILlmarti, W. W. \& Wooldridge, C. E. 1962 J. Fluid Mech. 14, 187.

Willmakth, W. W. \& Roos, F. W. 1965 J. Fluid Mech. 22, 81.

WILLS, J. A. B. 1970 J. Fluid Mech. 45, 65. 